\title{
18. BIOSTRATIGRAPHY OF UPPER CRETACEOUS AND PALEOGENE CALCAREOUS NANNOFOSSILS FROM LEG 123, NORTHEASTERN INDIAN OCEAN ${ }^{1}$
}

\author{
Michael J. Moran²
}

\begin{abstract}
Two of five holes drilled at two separate sites during Leg 123 of the Ocean Drilling Program intersected thick and relatively complete sections of Upper Cretaceous-Paleogene nannofossiliferous sediments. Although dominated by turbidite deposition in the upper part, Hole $765 \mathrm{C}$ contains a thick and relatively complete Albian-Oligocene section, including a particularly thick Aptian interval, with abundant and fairly well-preserved nannofossils. Several unconformities are confidently interpreted in this section that span much of the Santonian, late Campanian, Maestrichtian, late Eocene, and early Oligocene. Hole 766A contains a thick and relatively complete Albian-lower Eocene section having generally abundant and well-preserved nannofossils. Several unconformities also have been identified in this section that span much of the Coniacian, early Campanian, Maestrichtian, and late Eocene through early Pliocene. The chronostratigraphic position and length of all these unconformities may have considerable significance for reconstructing the sedimentary history and for interpreting the paleoceanography of this region.

A particularly thick section of upper Paleocene-lower Eocene sediments, including a complete record across the Paleocene/Eocene boundary, also was cored in Hole 766A that contains abundant and diverse nannofossil assemblages. Although assemblages from this section were correlated successfully using a standard low-latitude zonation, difficulties were encountered that reduced biostratigraphic resolution. Several lines of evidence suggest a mid-latitude position for Site 766 during this time, including (1) high assemblage diversity characteristic of mid-latitude zones of upwelling and (2) absence of certain ecologically controlled markers found only in low latitudes.
\end{abstract}

\section{INTRODUCTION}

During Leg 123 of the Ocean Drilling Program, scientists drilled five holes at two separate sites in the northeastern Indian Ocean, adjacent to the northwestern shelf region of Australia, from September through October 1988 (Fig. 1). This cruise, which represented a combined effort with Leg 122 scientists, attempted to explore and better understand the paleoceanographic history of the northwestern margin of Australia from Exmouth to Scott plateaus. This margin is thought to represent one of the oldest continent/ocean boundaries on Earth (Ludden, Gradstein, et al., 1990). The primary objectives of Leg 123 involved determining events in the final stages of rifting of the Exmouth Plateau and determining when volcanic basement and early oceanic sediments in the Argo Abyssal Plain were formed. The presence of generally abundant and well-preserved calcareous nannofossil assemblages in much of the Upper Cretaceous through Paleogene sediments from these holes provided the impetus for this study, which has yielded an accurate and detailed temporal framework for ascertaining the nature and timing of post-rifting events and for reconstructing the paleoceanography of this area, as well as identifying unconformities for interpreting regional sedimentary history.

Site 765 is located in the southern Argo Abyssal Plain at $15^{\circ} 58.41^{\prime} \mathrm{S}$ and $117^{\circ} 34.495^{\prime} \mathrm{E}$ in a water depth of $5728.2 \mathrm{~m}$ (Fig. 1). This site is located over what was thought to be the oldest oceanic crust in the Indian Ocean. Four holes were drilled; in the first three, sedimentary sections were cored. Hole $765 \mathrm{~A}$ was presumed to have missed the mud line during initial entry and was abandoned after the first core. Hole $765 \mathrm{~B}$ was drilled to a total

\footnotetext{
${ }^{1}$ Gradstein, F. M., Ludden, J. N., et al., 1992. Proc. ODP. Sci. Results, 123: College Station, TX (Ocean Drilling Program).

Department of Geology, University of Papua New Guinea, Port Moresby, Papua New Guinea.
}

depth of $386.3 \mathrm{~m}$ using both the advanced hydraulic piston corer (APC) and extended-core barrel (XCB) methods for maximum recovery. A Quaternary to middle Miocene section was recovered. Problems with recovery and penetration forced the scientists to drill a new hole (Hole 765C), which was begun at $350.2 \mathrm{mbsf}$ using the rotary-core barrel (RCB) method. This hole was penetrated to a total depth of $964 \mathrm{mbsf}$, which included approximately $30 \mathrm{~m}$ of basaltic basement. Preliminary shipboard biostratigraphy indicated that this hole intersected a middle Miocene to Berriasian section (Ludden, Gradstein, et al., 1990). The Aptian-Oligocene portion of this sequence, which spanned Cores 123-765C-34R through $-14 \mathrm{R}$, is examined here.

Site 766 is located on the western limit of the Exmouth Plateau at $19^{\circ} 55.985^{\prime} \mathrm{S}$ and $110^{\circ} 27.130^{\prime} \mathrm{E}$ in a water depth of $3997.5 \mathrm{~m}$ (Fig. 1). This site is positioned over the foot of the continental slope above the Gascoyne Abyssal Plain. Hole 766A was drilled at this site using only the RCB method and was penetrated a total of $527 \mathrm{mbsf}, 60.3 \mathrm{~m}$ of which consists of igneous material interpreted as basement. Shipboard biostratigraphy indicated the presence of a condensed Cenozoic section overlying a more complete Mesozoic section (Ludden, Gradstein, et al., 1990). The Albianlower Eocene portion, which spanned Cores 123-766A-21R through $-3 R$, is examined here.

\section{METHODS AND PROCEDURES}

Upper Cretaceous-Paleogene sediments from holes drilled at Sites 765 and 766 were examined for calcareous-nannofossil biostratigraphic information. Standard smear slides made from raw sediment samples were analyzed using the light microscope. The results are presented in Tables 1 through 4 . These tables graphically display information such as the abundance of nannofossils as a sedimentary component, relative abundance of individual nannofossil species, estimates of preservation of all nannofossil taxa, as well as stratigraphic distribution of nannofossil species and zonal assignment of samples. 


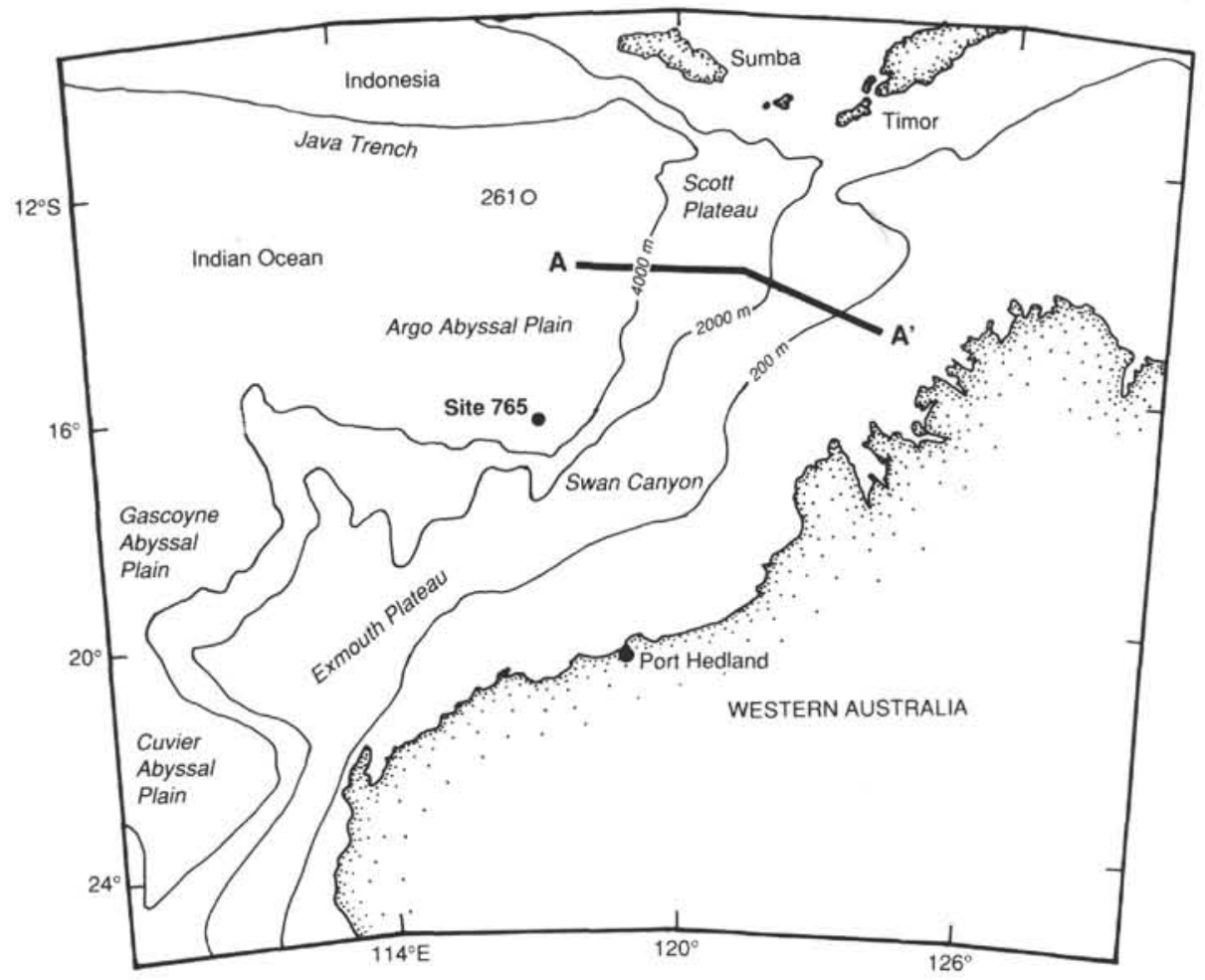

Figure 1. Location of sites drilled during Leg 123.

Letter designations in Tables 1 through 4 indicate the abundance of nannofossils as a sedimentary component and follow a method outlined by Watkins and Bowdler (1984). The criteria for letter designations are as follows:

$$
\begin{aligned}
& A=\text { abundant (nannofossils }>15 \% \text { of sediment) } \\
& C=\text { common (nannofossils } 5 \%-15 \% \text { of sediment) } \\
& F=\text { few (nannofossils } 1 \%-5 \% \text { of sediment) } \\
& R=\text { rare (nannofossils }<1 \% \text { of sediment) } \\
& B=\text { barren (no nannofossils in } 100 \text { fields of view at } 1500 \times \mathrm{X} \text { ) }
\end{aligned}
$$

Letter designations in Tables 1 through 4 that indicate the relative abundance of individual nannofossil species follow a method proposed by Hay (1970) and modified by Watkins and Bowdler (1984). The criteria for these letter designations are as follows:

$$
\begin{aligned}
& \mathrm{A}=\text { abundant }(1-10 \text { specimens per field of view at } 1500 \times) \\
& \mathrm{C}=\text { common }(1 \text { specimen per } 2-10 \text { fields of view at } 1500 \times) \\
& \mathrm{F}=\text { few }(1 \text { specimen per } 11-100 \text { fields of view at } 1500 \times) \\
& \mathrm{R}=\text { rare }(1 \text { specimen per } 101-1000 \text { fields of view at } 1500 \times) \\
& ?=\text { questionable presence of this taxa }
\end{aligned}
$$

Letter designations in Tables 1 through 4 that indicate estimates of average preservational state of all nannofossil taxa follow a method used by Moran and Watkins (1988). An estimate of the average preservational state of all nannofossils encountered is necessary because preservation can vary widely, not only between specimens from different taxonomic units, but also between individual specimens within a particular taxonomic unit. The criteria for letter designations are as follows:

$\mathrm{G}=\underset{\text { alteration) }}{\operatorname{good}(\operatorname{most} \text { specimens exhibit little or no secondary }}$
M = moderate (specimens exhibit significant secondary alteration in the form of overgrowth or dissolution but identification of species not impaired),

$\mathrm{P}=$ poor (specimens exhibit profound secondary alteration and identification of species is impaired but still possible).

A designation of "M-P" indicates an estimated preservational state intermediate between the two end components.

The biostratigraphic zonation scheme and zonal code numbers used for correlation of Cretaceous material follows that of Sissingh (1977), with the modifications of additional subdivision by alternate biohorizons, as suggested by Perch-Nielsen (1979; 1985), for greater biostratigraphic resolution. Here, the zonal code numbers of Sissingh (1977) are preceded by the abbreviation CC. This zonation scheme has proven most accurate and effective for worldwide low-latitude correlation of Cretaceous sediments. The Cenozoic section is correlated using the zonation scheme and zonal code numbers of Okada and Bukry (1980) because this method yields the highest resolution and greatest accuracy for worldwide, low-latitude correlation.

\section{RESULTS}

\section{Site 765}

Shipboard sedimentologic evidence from the section drilled at Hole $765 \mathrm{C}$ indicated that the Aptian-Paleogene portion (part of lithologic Units III and IV; Cores 123-765C-34R through -14R) consists lithologically of dominant hemipelagic clays and claystones and minor calcareous turbidites as well as other redeposited calcareous sediment (Ludden, Gradstein, et al., 1990). As a result of the minor effect of turbidity currents in the deposition of sediments in this interval, nannofossils exhibit little evidence of 
reworking, as opposed to those found in the Neogene section, where turbidity currents are thought to dominate the depositional environment (Ludden, Gradstein, et al., 1990). In addition, nannofossil assemblages in the Upper Cretaceous-Paleogene interval of Hole $765 \mathrm{C}$ are fairly well preserved, high in diversity, and nannofossils are common to abundant as a sedimentary component (Tables 1 and 2). These factors, along with good recovery for this interval $(55.9 \%)$ and a reasonably complete stratigraphic record, allowed for an accurate and high-resolution biostratigraphic correlation and for establishment of a useful temporal framework for this hole.

At the bottom of the studied interval, Sample 123-765C-34R$\mathrm{CC}$ has been placed in Subzone CC7a (Chiastozygus litterarius Zone) based on the presence of $C$. litterarius and Rhagodiscus angustus and is Aptian in age (Fig. 2). Reworking of Jurassic material is evident with the presence of Parhabdolithus liasicus. Sample 123-765C-33R-CC is barren of nannofossils. Sediments between Sample 123-765C-33R-1, 62-63 cm, and -32R-3, 63-64 $\mathrm{cm}$, have been placed in Subzone CC7b based on the presence of the previously mentioned species and are also of Albian-Aptian age. The Aptian/Albian boundary falls within Subzone CC7b (Chiastozygus litterarius Zone) and thus occurs within this interval. The subzonal designation $\mathrm{CC} 7 \mathrm{~b}$ was given based on the presence of Hayesites albiensis and Eprolithus floralis in the absence of Micrantholithus obtusus. Samples 123-765C-32R-2, $67-69 \mathrm{~cm}$, and $-32 \mathrm{R}-1,64-65 \mathrm{~cm}$, are barren of nannofossils. Sediments of Albian age continue in Samples 123-765C-31R-CC through $-26 \mathrm{R}-3,118-119 \mathrm{~cm}$, and were placed in Zone CC8 (Prediscosphaera columnata Zone) based on the presence of $P$. columnata and are of Albian age. A subdivision of Subzone CC8b occurs in Sample 123-765C-28R-CC based on the first appearance of Tranolithus phacelosus.

The first appearance of Eiffellithus turriseiffeli in Sample 123-765C-26R-3, 32-33 cm, marks the base of Zone CC9 (Eiffellithus turriseiffeli Zone), which continues through Sample 123$765 \mathrm{C}-26 \mathrm{R}-3,25-26 \mathrm{~cm}$, and indicates an age of late Albian. The next productive samples (Sample 123-765C-25R-CC as well as 123-765C-25R-5, 35-36 cm) were placed in Zone CC11 (Quadrum gartneri Zone) based on the presence of $Q$. gartneri, $E$. turriseiffeli, and E. floralis and is early to middle Turonian in age. Because of sampling restrictions, the completeness of the Cenomanian section and the Cenomanian/Turonian boundary is uncertain; Zone CC10 may be present. However, the small amount of material between Samples 123-765C-26R-3, 25-26 cm, and $-25 \mathrm{R}-\mathrm{CC}(3.25 \mathrm{~m})$ suggests a condensed or partially missing Cenomanian section.

The next available sample (Sample 123-765C-25R-3, 15-19 $\mathrm{cm}$ ) contains Eiffellithus eximius, placing it within Zone CC12 (Lucianorhabdus maleformis Zone), and yielded an age of late Turonian-early Coniacian. Sediments of Coniacian age were also found in Sample 123-765C-25R-2, 48-49 cm, and were assigned to Zone CC13 (Marthasterites furcatus Zone) based on the presence of $M$. furcatus. Sediments in Samples 123-765C-25R-1, $33-35 \mathrm{~cm}$, through $-4 \mathrm{R}-4,41-43 \mathrm{~cm}$, were placed in Zone $\mathrm{CC} 17$

\begin{tabular}{|c|c|c|c|c|}
\hline & & & $\begin{array}{l}\text { Argo Abyssal } \\
\text { Hole } 765 \mathrm{C}\end{array}$ & $\begin{array}{c}\text { Exmouth Plateau } \\
\text { Hole } 766 \mathrm{~A}\end{array}$ \\
\hline \multirow{4}{*}{ Maastrichtian } & N. frequens & cc26 & & $10 \mathrm{R}-2,114 \rightarrow 10 \mathrm{R}-3,13$ \\
\hline & A. cymbiformis & $\mathrm{cc} 25$ & & \\
\hline & R. levis & $\mathrm{cc} 24$ & & \\
\hline & T. phacelosus & $\mathrm{cc} 23$ & $23 R-3,93 \rightarrow 23 R-4$ & $10 \mathrm{R}, \mathrm{CC}$ \\
\hline \multirow{6}{*}{ Campanian } & Q. trifidum & $\mathrm{cc} 22$ & & $\begin{array}{c}11 R \\
12 R-1,114 \rightarrow 12-2,27\end{array}$ \\
\hline & Q. sissinghi & $\mathrm{CC} 21$ & & $12 \mathrm{R}, \mathrm{CC}$ \\
\hline & C. aculeus & $\mathrm{Cc} 20$ & $23 \mathrm{R}, \mathrm{CC}$ & ): \\
\hline & C. ovalis & CC19 & $24 \mathrm{R}-1,36 \rightarrow 24 \mathrm{R}-3,95$ & $13 R-1,38 \rightarrow 13-2,30$ \\
\hline & A. parcus & cc18 & & \\
\hline & c. obscurus & $\mathrm{CC}_{17}$ & $24 \mathrm{R}-4,41 \rightarrow 25 \mathrm{R}-1,34$ & \\
\hline \multirow[b]{2}{*}{ Santonian } & L. cayeuxii & $\mathrm{CC} 16$ & & \multirow{2}{*}{$13 \mathrm{R}-3,69 \rightarrow 14 \mathrm{R}-1,46$} \\
\hline & R. anthophorus & CC15 & & \\
\hline \multirow[b]{2}{*}{ Coniacian } & M. decussata & CC14 & 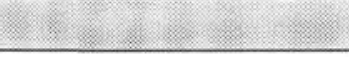 & : \\
\hline & M. furcatus & $\mathrm{CC}_{13}$ & $25 R-2,48$ & $14 \mathrm{R}-2,23$ \\
\hline \multirow[b]{2}{*}{ Turonian } & L. maleformis & $\mathrm{CC}_{12}$ & $25 \mathrm{R}-3,17$ & $14 \mathrm{R}-3,23$ \\
\hline & Q. gartneri & CC11 & $25 \mathrm{R}, \mathrm{CC}$ & $14 \mathrm{R}-4 \rightarrow 14, \mathrm{CC}$ \\
\hline \multirow[t]{2}{*}{ Cenomanian } & M. decoratus & CC10 & & \\
\hline & E. turriseiffelii & $\operatorname{ccs}$ & $26 \mathrm{R}-3,25 \rightarrow 26 \mathrm{R}-3,33$ & $15 R, C C \rightarrow 17 R-4,82$ \\
\hline Albian & P. columnata & $\mathrm{cc} 8$ & $\begin{array}{c}26-3,118 \rightarrow 28 R \\
29 R \rightarrow 31 R \\
\end{array}$ & $17 R, C C \rightarrow 21 R$ \\
\hline Aptian & C. litterarius & $\operatorname{cc} 7$ & $\begin{array}{c}32 \mathrm{R}-3,63 \rightarrow 33 \mathrm{R}-1,63 \\
34 \mathrm{R}\end{array}$ & 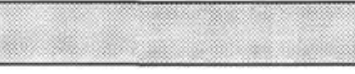 \\
\hline
\end{tabular}

Figure 2. Distribution of Cretaceous sediments from Leg 123 based on calcareous nannofossil biostratigraphy. 
Table 1. Cretaceous calcareous nannofossils from Site 765.

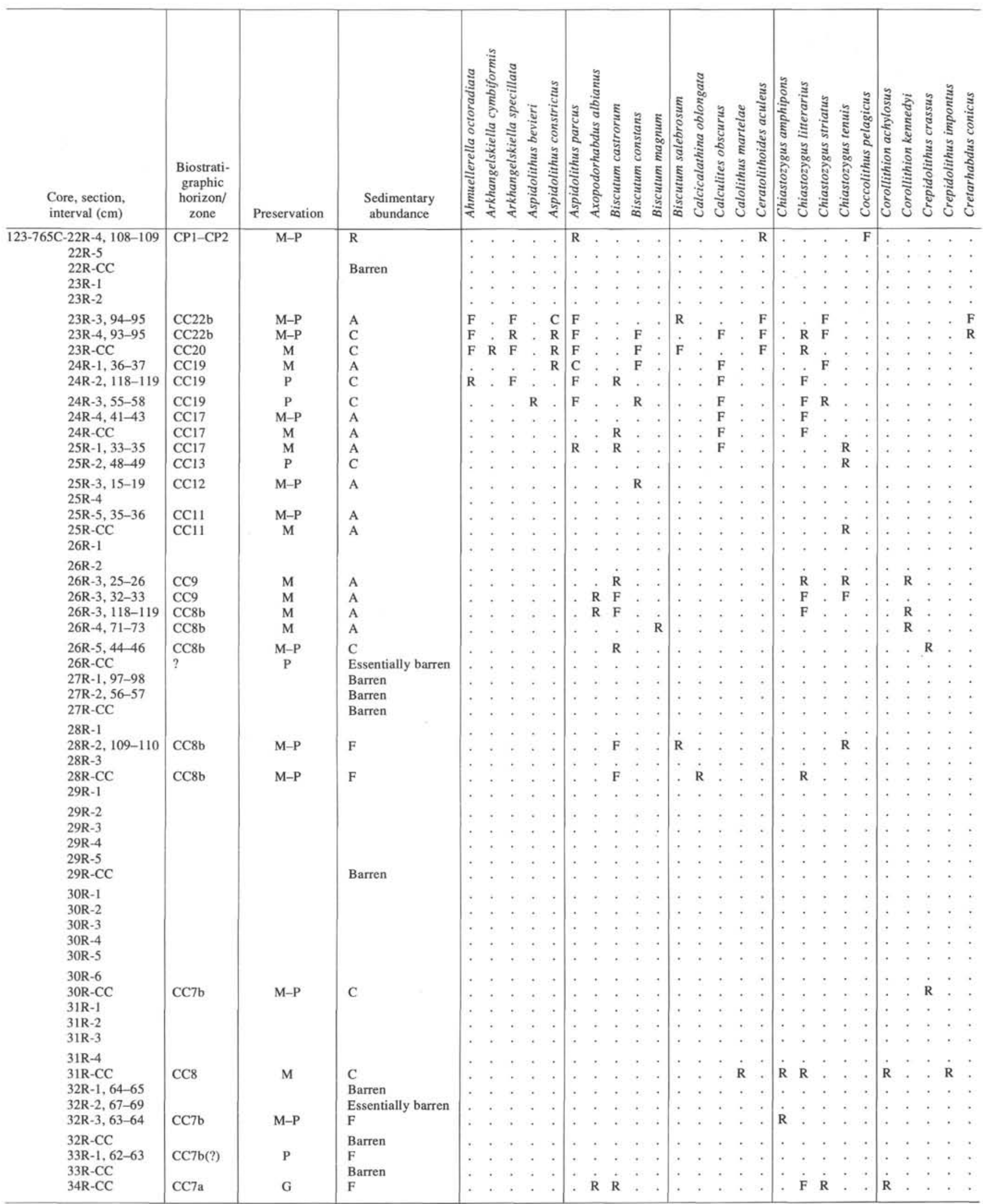

Key to symbols: ? = implied presence; $\mathrm{RE}=$ Reworked; $\mathrm{VR}=$ Very rare; $\mathrm{R}=$ Rare; $\mathrm{F}=\mathrm{Few} ; \mathrm{C}=\mathrm{Common} ; \mathrm{A}=\mathrm{Abundant} ; \mathrm{VA}=\mathrm{Very}$ abundant; ? $=\mathrm{Questionably}$ present; $=$ Not present. 


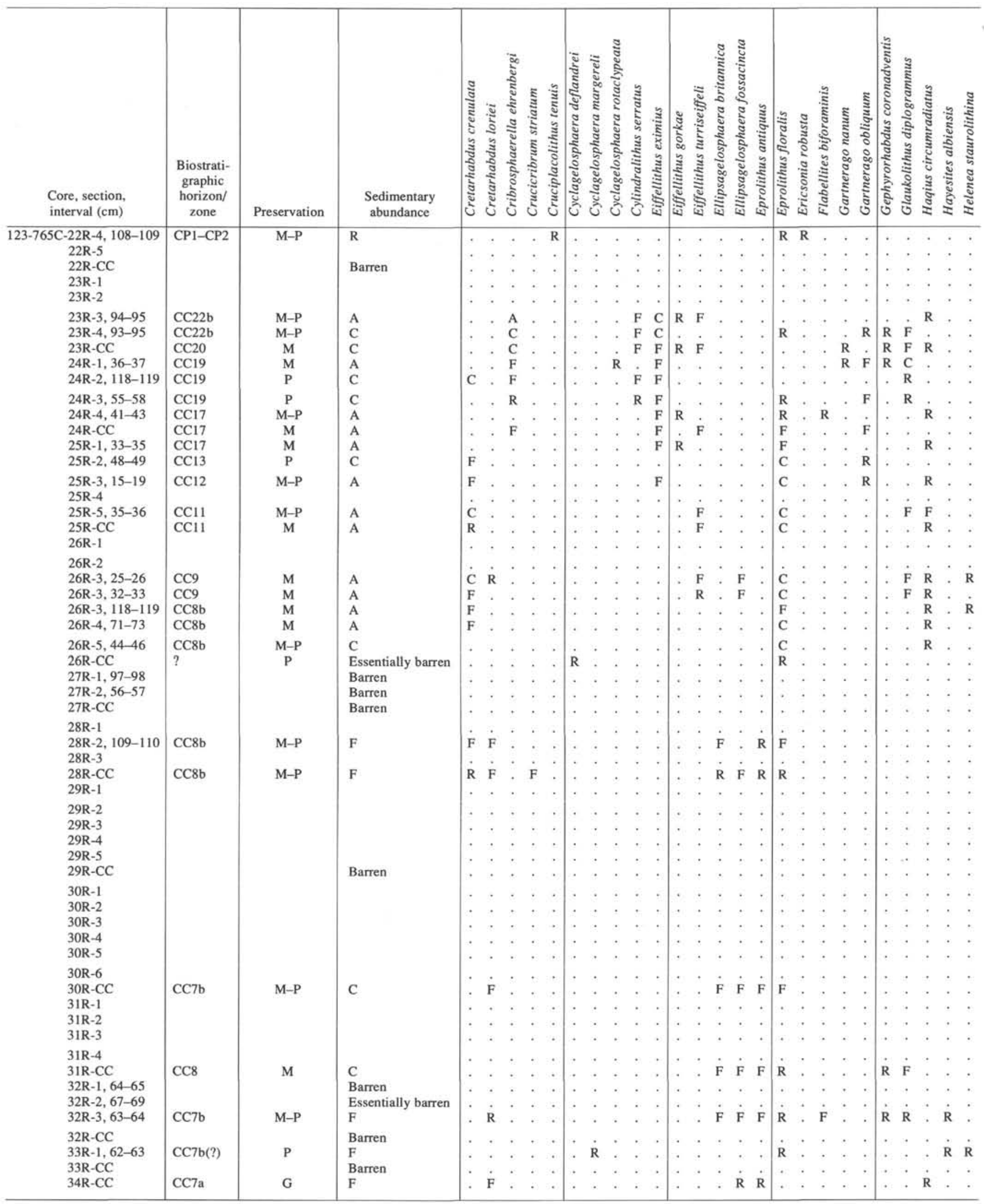


Table 1 (continued).

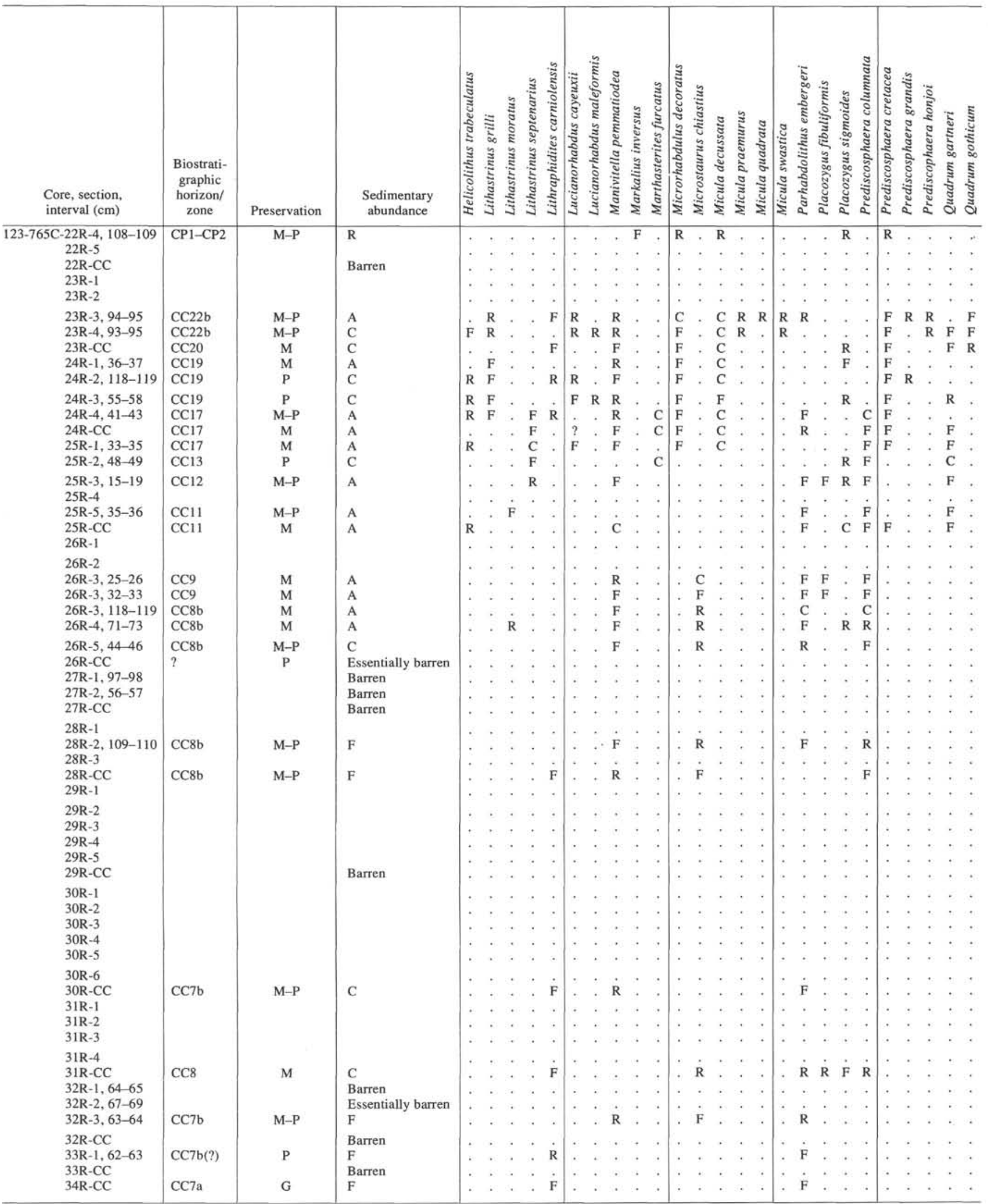


Table 1 (continued).

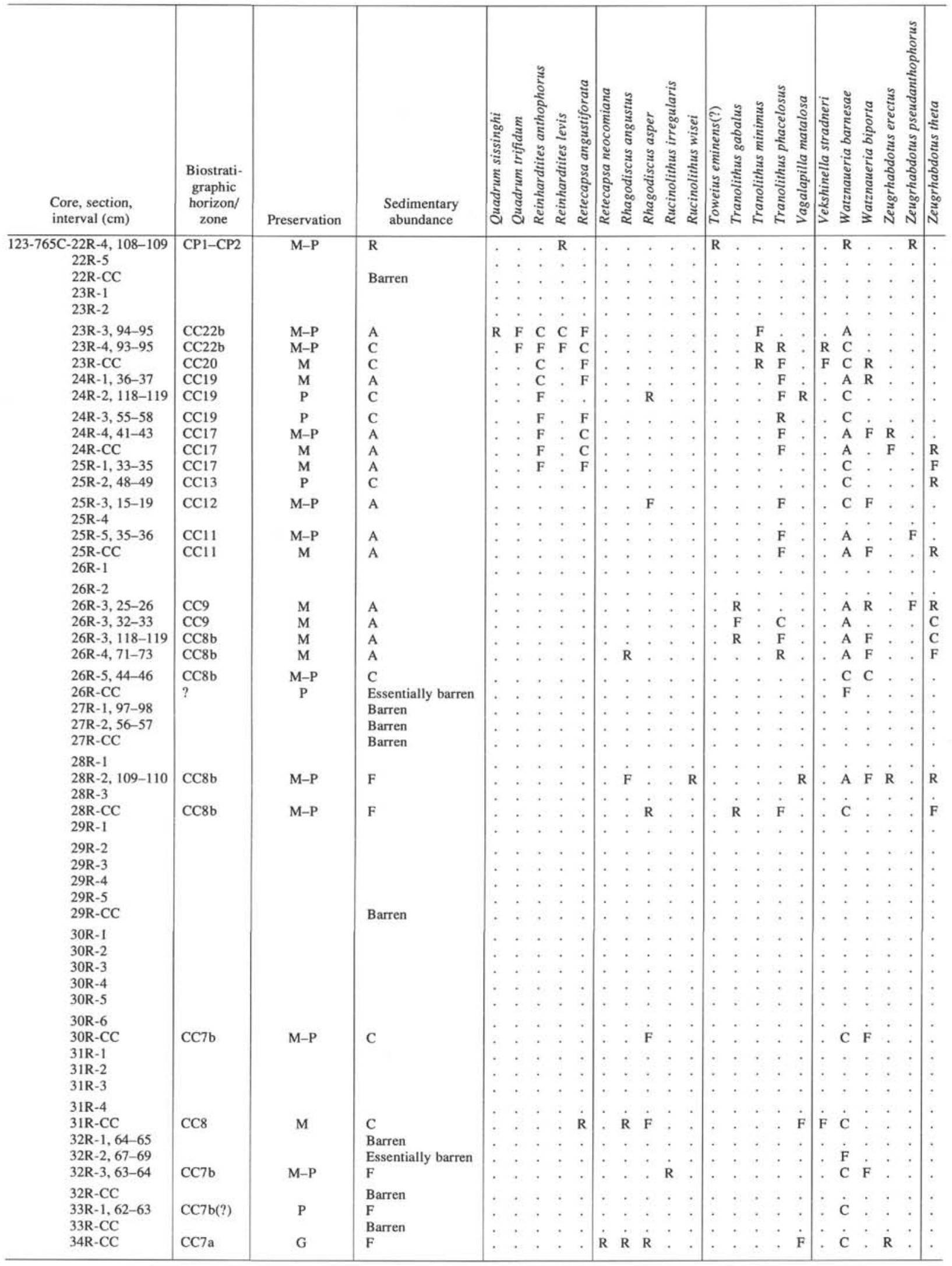


Table 2. Paleogene calcareous nannofossils from Site 765.

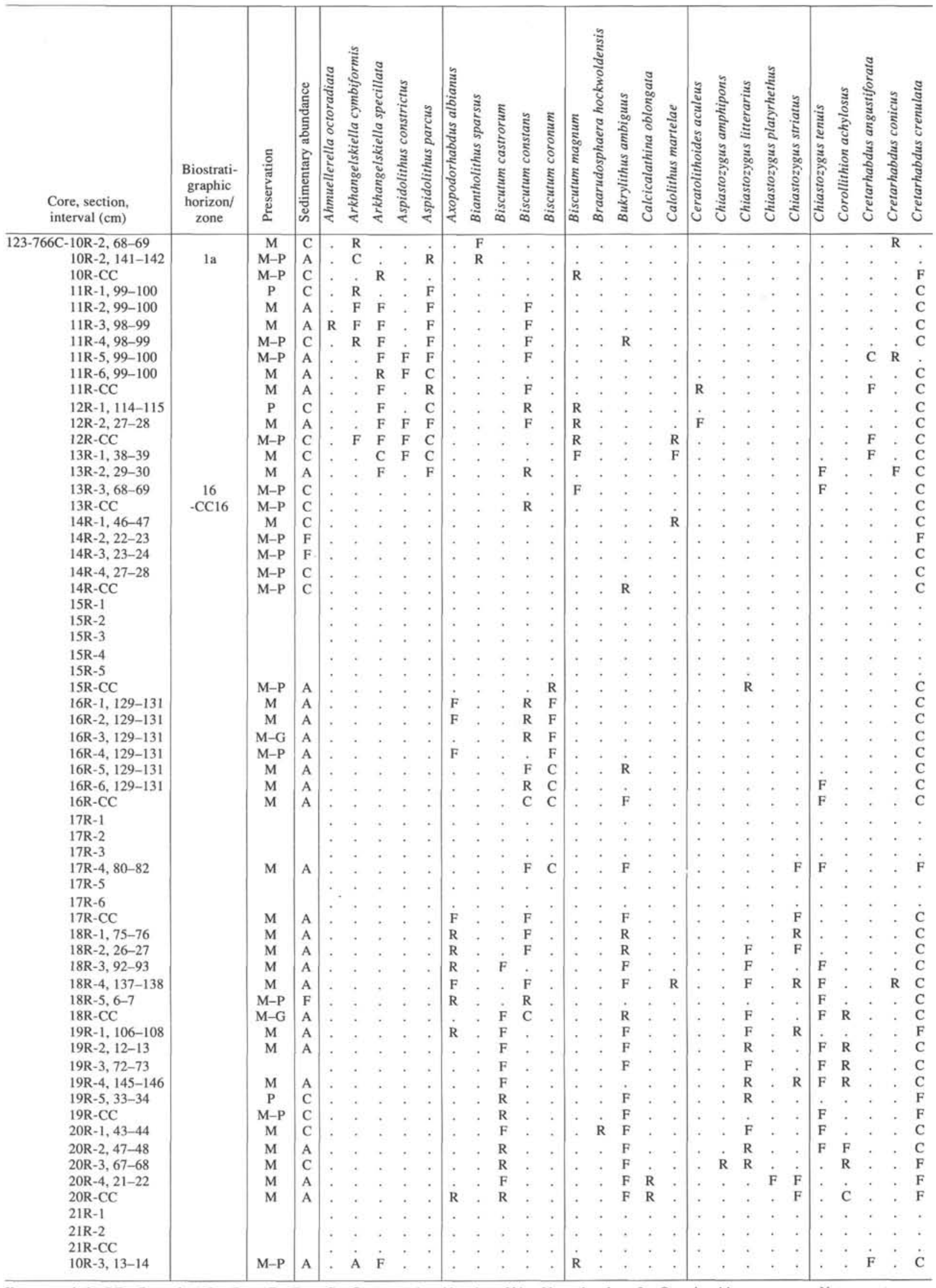

Key to symbols: $\mathrm{RE}=$ Reworked; $\mathrm{R}=\mathrm{Rare} ; \mathrm{F}=\mathrm{Few} ; \mathrm{C}=$ Common; $\mathrm{A}=$ Abundant; $\mathrm{VA}=$ Very abundant; $?=$ Questionable presence; . = Not present. 
Table 2 (continued).

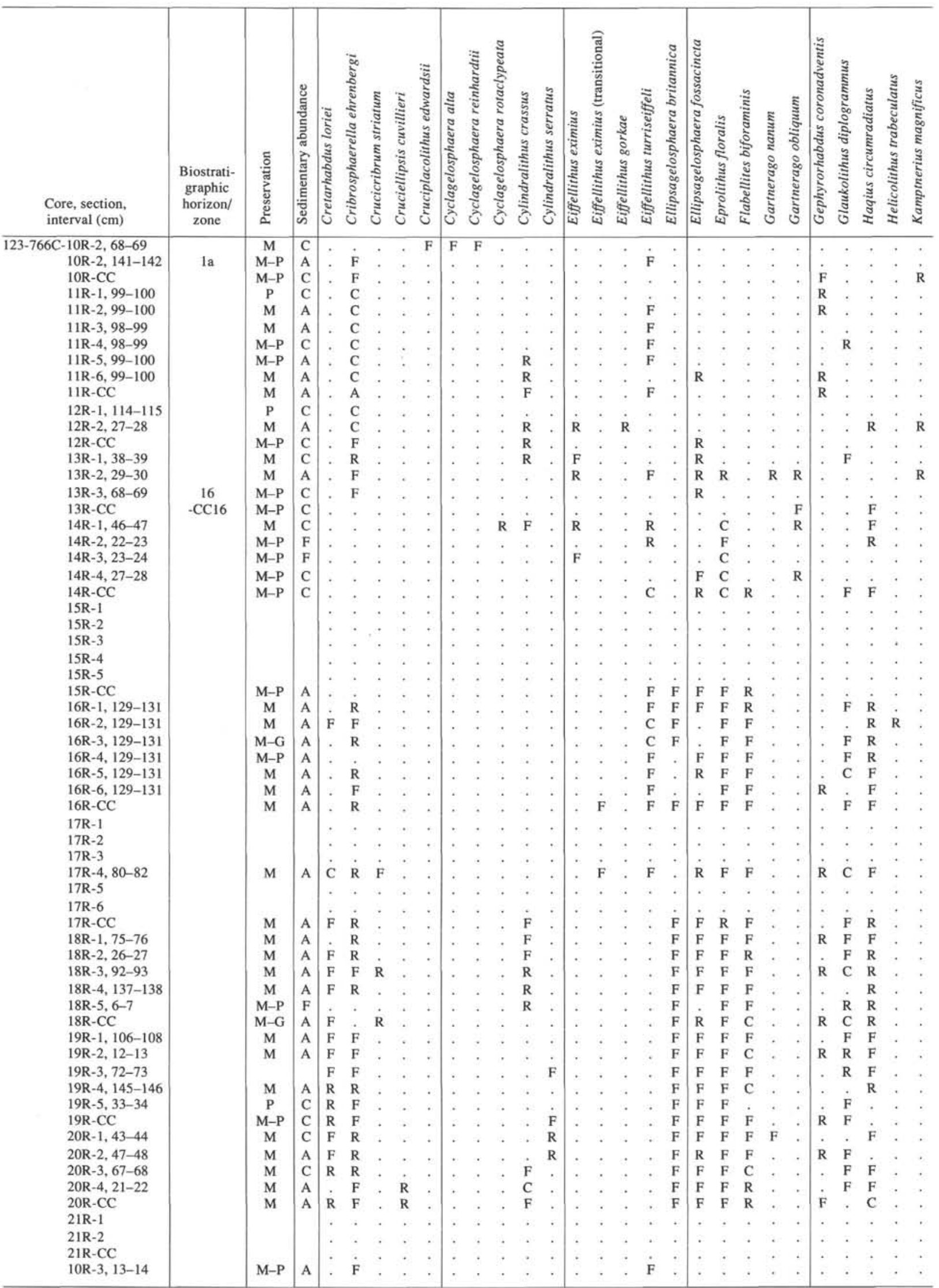


Table 2 (continued).

\begin{tabular}{|c|c|c|c|c|c|c|c|c|c|c|c|c|c|c|c|c|c|c|c|c|c|c|c|c|c|c|c|c|}
\hline $\begin{array}{l}\text { Core, section, } \\
\text { interval }(\mathrm{cm})\end{array}$ & $\begin{array}{c}\text { Biostrati- } \\
\text { graphic } \\
\text { horizon/ } \\
\text { zone }\end{array}$ & 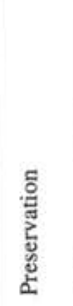 & 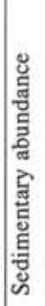 & 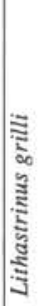 & 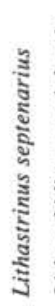 & 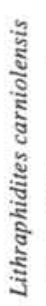 & 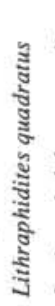 & 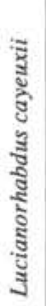 & 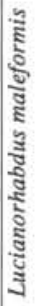 & 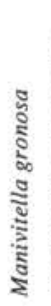 & 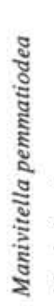 & 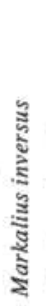 & 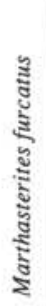 & 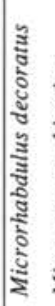 & 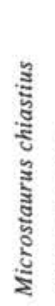 & 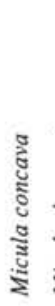 & 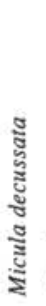 & 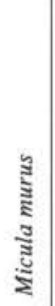 & 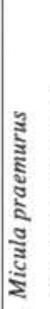 & 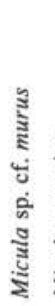 & 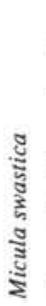 & 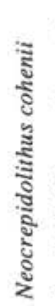 & 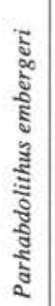 & 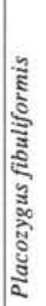 & 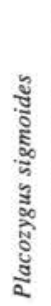 & 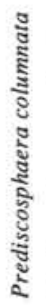 & 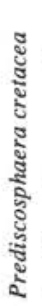 & 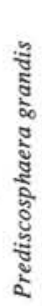 \\
\hline $123-766 \mathrm{C}-10 \mathrm{R}-2,68-69$ & & M & $\mathrm{C}$ & . & . & . & $\mathbf{R}$ & . & . & . & . & $\mathrm{C}$ & . & . & . & . & $\mathrm{R}$ & $\mathrm{R}$ & . & . & . & $\mathrm{F}$ & . & . & A & . & $\mathbf{R}$ & . \\
\hline $10 \mathrm{R}-2,141-142$ & la & $\mathrm{M}-\mathrm{P}$ & A & . & . & $\mathrm{F}$ & . & . & . & . & $\mathbf{R}$ & $\mathrm{F}$ & . & . & . & . & $\mathrm{A}$ & $\mathrm{F}$ & . & . & $\mathbf{R}$ & . & . & . & . & . & $\mathrm{C}$ & F \\
\hline 10R-CC & & M-P & C & . & . & $\mathrm{F}$ & . & . & . & . & $\mathrm{F}$ & . & . & $\mathrm{F}$ & . & . & $\mathrm{C}$ & . & . & . & . & . & . & . & F & . & $\mathrm{F}$ & $\therefore$ \\
\hline $11 \mathrm{R}-1,99-100$ & & $\mathrm{P}$ & $\mathrm{C}$ & . & . & $\mathrm{F}$ & . & . & . & . & . & . & . & . & . & . & C & . & . & . & . & . & . & . & F & . & $\mathrm{F}$ & F \\
\hline $1 \mathrm{IR}-2,99-100$ & & $\mathrm{M}$ & A & . & . & $\mathrm{C}$ & . & . & . & . & $\mathbf{R}$ & . & . & $\mathrm{F}$ & . & . & A & . & . & $\mathbf{R}$ & $\mathrm{F}$ & . & . & . & $\mathrm{F}$ & $\mathrm{F}$ & $\mathrm{F}$ & F \\
\hline $11 R-3,98-99$ & & M & A & . & . & $\mathrm{C}$ & . & . & . & . & $\mathrm{F}$ & . & . & $\mathrm{F}$ & . & . & C & . & . & . & $\mathbf{R}$ & . & . & . & $\mathbf{F}$ & $\mathrm{F}$ & $\mathrm{C}$ & $\mathrm{F}$ \\
\hline $11 R-4,98-99$ & & M-P & $\mathrm{C}$ & . & . & $\mathrm{C}$ & . & . & . & . & $\mathrm{F}$ & . & . & F & . & F & C & . & . & . & $\mathbf{R}$ & . & . & . & . & $\mathrm{F}$ & . & $\mathrm{F}$ \\
\hline $11 R-5,99-100$ & & M-P & A & . & . & $\mathrm{F}$ & . & . & . & . & $\mathbf{R}$ & , & . & $\mathrm{F}$ & . & $\mathrm{F}$ & C & . & F & . & $\mathbf{R}$ & . & . & . & . & $\mathrm{F}$ & $\dot{.}$ & $\mathrm{F}$ \\
\hline $11 \mathrm{R}-6,99-100$ & & M & A & . & . &.$F$ & . & . & . & . & $\mathbf{R}$ & . & . & F & . & $\mathrm{C}$ & C & . & F & . & $\mathbf{R}$ & . & . & . & . & . & $\mathrm{F}$ & $\mathrm{F}$ \\
\hline $11 \mathrm{R}-\mathrm{CC}$ & & M & A & . & . & C & $\mathbf{R}$ & . & . & . & . & . & . & $\mathrm{F}$ & . & $\mathrm{F}$ & C & . & . & . & . & . & . & . & . & $\mathrm{F}$ & $\mathrm{C}$ & $\mathbf{R}$ \\
\hline $12 \mathrm{R}-1,114-115$ & & $\mathrm{P}$ & $\mathrm{C}$ & . & . & $\mathrm{F}$ & . & . & . & . & $\mathbf{R}$ & $\mathbf{R}$ & . & $\mathbf{R}$ & . & . & C & . & . & . & . & . & . & . & . & $\mathrm{F}$ & F & . \\
\hline $12 \mathrm{R}-2,27-28$ & & $\mathrm{M}$ & A & . & . & $\mathrm{C}$ & . & . & . & . & $\mathbf{R}$ & . & . & $\mathrm{F}$ & . & . & C & . & . & . & $\mathrm{F}$ & . & . & . & . & $\mathrm{F}$ & C & F \\
\hline $12 \mathrm{R}-\mathrm{CC}$ & & M-P & C & . & . & $\mathrm{F}$ & . & . & . & . & $\mathbf{R}$ & , & . & . & . & $\mathrm{C}$ & $\mathrm{C}$ & . & . & . & $\dot{\therefore}$ & . & $\therefore$ & $\dot{0}$ & . & $\dot{\dot{ }}$ & $\mathrm{F}$ & . \\
\hline $13 \mathrm{R}-1,38-39$ & & M & C & . & . & $\mathrm{F}$ & . & $\mathrm{F}$ & . & $\mathbf{R}$ & $\mathbf{R}$ & . & . & $\mathbf{R}$ & . & $\dot{.}$ & C & . & . & . & $\mathbf{R}$ & . & $\mathrm{F}$ & F & . & $\mathrm{C}$ & C & $\therefore$ \\
\hline $13 R-2,29-30$ & & M & A & . & . & $\mathrm{C}$ & . & $\mathrm{R}$ & . & . & . & $\mathbf{R}$ & . & $\mathrm{R}$ & . & $\mathrm{F}$ & $\mathrm{C}$ & . & . & . & . & . & . & . & . & $\mathrm{F}$ & $\cdot$ & $\mathrm{F}$ \\
\hline $13 R-3,68-69$ & 16 & M-P & C & $\mathbf{R}$ & $\mathrm{F}$ & $\mathrm{C}$ & . & . & . & . & $\mathrm{F}$ & . & C & $\mathbf{R}$ & . & . & C & . & . & . & . & . & . & . & . & F & . & . \\
\hline $13 \mathrm{R}-\mathrm{CC}$ & $-\mathrm{CC} 16$ & M-P & $\mathrm{C}$ & . & . & $\mathrm{C}$ & . & . & . & $\mathrm{R}$ & $\mathrm{F}$ & . & A & . & . & $\mathrm{F}$ & C & . & . & . & . & . & . & . & . & $\mathrm{F}$ & . & , \\
\hline $14 \mathrm{R}-1,46-47$ & & M & C & $\mathbf{R}$ & $\mathrm{F}$ & $\mathrm{F}$ & . & . & $?$ & $\mathrm{R}$ & $\mathrm{F}$ & $\mathbf{R}$ & A & . & . & $\mathrm{F}$ & $\mathrm{F}$ & . & . & . & . & . & . & . & . & C & . & . \\
\hline $14 \mathrm{R}-2,22-23$ & & $\mathrm{M}-\mathrm{P}$ & $\mathrm{F}$ & . & $\mathrm{R}$ & $\mathrm{F}$ & . & . & $?$ & $\mathbf{R}$ & $\mathrm{F}$ & . & $\mathrm{F}$ & . & . & . & . & . & . & . & . & . & . & . & . & $\mathrm{F}$ & . & . \\
\hline $14 \mathrm{R}-3,23-24$ & & M-P & $\mathrm{F}$ & . & . & $\mathrm{F}$ & . & . & . & $\mathrm{F}$ & $\mathrm{F}$ & . & . & . & . & . & . & . & . & . & . & . & $\mathbf{R}$ & . & . & $\mathrm{C}$ & $\mathrm{F}$ & . \\
\hline $14 \mathrm{R}-4,27-28$ & & M-P & $\mathrm{C}$ & . & . & $\mathrm{F}$ & . & . & . & $\mathrm{F}$ & $\mathrm{F}$ & . & . & . & . & . & . & . & . & . & . & . & . & $\mathrm{F}$ & . & $\mathrm{C}$ & . & . \\
\hline $14 \mathrm{R}-\mathrm{CC}$ & & M-P & C & . & . & $\mathrm{F}$ & . & . & . & . & $\mathrm{F}$ & . & . & . & . & . & . & . & . & . & . & . & $\mathrm{F}$ & F & . & $\mathrm{C}$ & . & . \\
\hline $15 \mathrm{R}-1$ & & & & . & . & . & . & . & . & . & . & . & . & . & . & . & . & . & . & . & . & . & $\cdot$ & . & . & - & . & . \\
\hline $15 R-2$ & & & & , & . & . & . & . & . & . & . & . & . & . & . & . & . & . & . & . & . & . & . & . & . & - & . & . \\
\hline $15 R-3$ & & & & . & . & . & . & . & . & . & . & . & . & . & . & . & . & . & . & . & . & . & . & . & . & . & . & . \\
\hline $15 R-4$ & & & & . & . & . & . & . & . & . & . & . & . & . & . & . & . & . & . & . & . & . & . & . & . & . & . & . \\
\hline $15 R-5$ & & & & . & . & . & . & . & . & . & . & . & . & . & . & . & . & . & . & . & . & . & $\dot{0}$ & . & . & $\dot{8}$ & . & . \\
\hline $15 \mathrm{R}-\mathrm{CC}$ & & M-P & A & . & . & $\dot{.}$ & . & . & . & $\mathrm{F}$ & $\mathrm{C}$ & . & . & . & $\mathrm{F}$ & . & . & . & . & . & . & . & $\mathrm{F}$ & . & . & C & . & . \\
\hline $16 \mathrm{R}-1,129-131$ & & M & A & . & . & $\mathrm{R}$ & . & . & . & $\mathrm{F}$ & $\mathrm{F}$ & . & . & . & $\mathrm{F}$ & . & . & . & . & . & . & . & C & . & . & $\mathrm{F}$ & . & . \\
\hline $16 \mathrm{R}-2,129-131$ & & M & A & . & . & $\mathbf{R}$ & . & . & . & $\mathbf{R}$ & $\mathrm{C}$ & . & . & . & $\mathrm{F}$ & . & . & . & . & . & . & . & $\mathrm{F}$ & . & . & $\mathrm{F}$ & . & . \\
\hline $16 \mathrm{R}-3,129-131$ & & $M-G$ & A & . & . & $\mathrm{F}$ & . & . & . & $\mathrm{F}$ & C & . & . & . & C & . & . & . & . & . & . & . & $\mathrm{F}$ & . & . & $\mathrm{C}$ & . & . \\
\hline $16 \mathrm{R}-4,129-131$ & & $\mathrm{M}-\mathrm{P}$ & A & . & . & . & . & . & . & . & $\mathrm{F}$ & . & . & . & $\mathrm{F}$ & . & . & . & . & . & . & . & $\mathrm{F}$ & . & . & $\mathrm{F}$ & . & . \\
\hline $16 \mathrm{R}-5,129-131$ & & M & A & . & . & $\mathrm{F}$ & . & . & . & $\mathrm{F}$ & $\mathrm{F}$ & . & . & , & . & . & . & . & . & . & . & . & $\mathrm{F}$ & . & . & F & . & . \\
\hline $16 \mathrm{R}-6,129-131$ & & M & A & . & . & . & . & . & . & . & $\mathrm{C}$ & . & . & . & $\mathrm{F}$ & . & . & . & . & . & . & . & $\mathrm{F}$ & . & . & $\mathrm{F}$ & . & . \\
\hline 16R-CC & & M & A & . & . & $\mathbf{R}$ & . & . & . & $F$ & $\mathrm{~F}$ & . & . & . & $\mathrm{F}$ & . & . & . & . & . & . & . & C & . & . & F & . & . \\
\hline $17 R-1$ & & & & . & . & . & . & . & . & . & . & . & . & . & . & . & . & . & . & . & . & . & . & . & . & . & . & . \\
\hline $17 \mathrm{R}-2$ & & & & . & . & . & . & . & . & . & . & . & . & . & . & . & . & . & . & . & . & . & . & . & . & - & . & . \\
\hline $17 \mathrm{R}-3$ & & & & . & . & . & . & . & . & . & . & . & . & . & . & . & . & . & . & . & . & . & . & . & . & . & . & . \\
\hline $17 R-4,80-82$ & & M & A & . & . & $\mathrm{F}$ & . & . & . & $\mathrm{F}$ & $\mathrm{F}$ & . & . & . & $\mathrm{C}$ & . & . & . & . & . & . & . & $\mathrm{F}$ & . & . & $\mathrm{F}$ & . & . \\
\hline $17 \mathrm{R}-5$ & & & & . & . & . & . & . & . & . & . & . & . & . & . & . & . & . & . & . & . & . & $\cdot$ & . & . & . & . & . \\
\hline $17 R-6$ & & & & . & . & . & . & . & . & . & . & . & . & . & . & . & . & . & . & . & . & . & . & . & . & - & . & . \\
\hline $17 \mathrm{R}-\mathrm{CC}$ & & M & A & . & . & F & . & . & . & $\mathrm{F}$ & $\mathrm{F}$ & . & . & . & $\mathrm{F}$ & . & . & . & . & . & . & . & $\mathrm{F}$ & . & . & $\mathbf{R}$ & . & . \\
\hline $18 \mathrm{R}-1,75-76$ & & M & A & . & . & $\mathrm{F}$ & . & . & . & $\mathrm{F}$ & $\mathrm{F}$ & . & . & . & $\mathrm{F}$ & . & . & . & . & . & . & . & $\mathrm{F}$ & . & . & $\mathbf{R}$ & . & . \\
\hline $18 \mathrm{R}-2,26-27$ & & M & A & . & . & $\mathrm{C}$ & . & . & . & $\mathbf{R}$ & $\mathrm{F}$ & . & . & . & $\mathrm{F}$ & . & . & . & . & . & . & . & $\mathrm{F}$ & . & . & $\mathrm{F}$ & . & . \\
\hline $18 \mathrm{R}-3,92-93$ & & M & A & . & . & $\mathrm{F}$ & . & . & . & $\mathbf{R}$ & . & . & . & . & $\mathrm{F}$ & . & . & . & . & . & . & . & $\mathrm{F}$ & . & . & $\mathrm{F}$ & . & . \\
\hline $18 R-4,137-138$ & & M & A & . & . & $\mathrm{C}$ & . & . & . & $\mathrm{R}$ & $\mathrm{F}$ & . & . & , & $\mathrm{F}$ & . & . & . & . & . & . & . & $\mathrm{F}$ & . & . & $\mathrm{F}$ & . & . \\
\hline $18 \mathrm{R}-5,6-7$ & & M-P & $\mathrm{F}$ & . & . & . & . & . & . & $\mathbf{R}$ & $\mathrm{F}$ & . & . & . & $\mathrm{F}$ & . & . & . & . & . & . & . & $\mathrm{F}$ & . & . & $\mathrm{F}$ & . & . \\
\hline 18R-CC & & M-G & A & . & . & $\mathrm{F}$ & . & . & . & $\mathbf{R}$ & $\mathrm{F}$ & . & . & . & $\mathrm{F}$ & . & . & . & . & . & . & . & $\mathrm{C}$ & $\mathrm{F}$ & . & $\mathrm{F}$ & . & . \\
\hline $19 \mathrm{R}-1,106-108$ & & M & A & . & . & C & . & . & . & $\mathrm{F}$ & $\mathrm{F}$ & . & . & . & $\mathrm{F}$ & . & . & . & . & . & . & . & $\mathrm{F}$ & . & . & $\mathbf{R}$ & . & . \\
\hline $19 \mathrm{R}-2,12-13$ & & $\mathrm{M}$ & A & . & . & $\mathrm{C}$ & . & . & . & $\mathrm{F}$ & $\mathrm{F}$ & . & . & . & $\mathbf{F}$ & . & . & . & . & . & . & . & $\mathrm{F}$ & . & . & $\mathrm{F}$ & . & . \\
\hline $19 \mathrm{R}-3,72-73$ & & & & . & . & $\mathrm{C}$ & . & . & . & $\mathrm{F}$ & $\mathrm{F}$ & . & . & . & $\mathrm{F}$ & . & . & . & . & . & . & . & $\mathrm{F}$ & . & . & $\mathbf{R}$ & . & . \\
\hline $19 \mathrm{R}-4,145-146$ & & M & A & . & . & $\mathrm{C}$ & . & . & . & $\mathrm{F}$ & $\mathrm{F}$ & . & . & . & $\mathrm{F}$ & . & . & . & . & . & . & . & $\mathrm{F}$ & . & . & $\mathrm{F}$ & . & . \\
\hline $19 \mathrm{R}-5,33-34$ & & $\mathrm{P}$ & C & . & . & $\mathrm{F}$ & . & . & . & . & $\mathrm{F}$ & . & . & . & $\mathrm{F}$ & . & . & . & . & . & . & . & C & . & . & $\therefore$ & . & . \\
\hline 19R-CC & & $\mathrm{M}-\mathrm{P}$ & C & . & . & $\mathrm{C}$ & . & . & . & $\mathrm{F}$ & $\mathrm{F}$ & , & . & . & $\mathrm{F}$ & . & . & . & . & . & . & . & $\mathrm{F}$ & . & . & $\mathbf{F}$ & . & . \\
\hline $20 \mathrm{R}-1,43-44$ & & M & $\mathrm{C}$ & . & . & $\mathrm{F}$ & . & . & . & $\mathrm{F}$ & $\mathbf{R}$ & . & . & . & $\mathbf{F}$ & . & . & . & . & . & . & . & $\mathrm{F}$ & . & . & $\mathrm{R}$ & . & . \\
\hline $20 \mathrm{R}-2,47-48$ & & M & A & . & , & C & . & , & . & $\mathrm{F}$ & $\mathrm{F}$ & . & . & $\cdot$ & $\mathrm{F}$ & . & . & . & . & . & . & . & $\mathrm{C}$ & . & . & $\mathrm{F}$ & . & . \\
\hline $20 R-3,67-68$ & & M & C & . & . & C & . & . & . & . & $\mathrm{R}$ & . & . & . & C & . & . & . & . & . & . & . & C & . & . & $\mathbf{R}$ & . & . \\
\hline $20 \mathrm{R}-4,21-22$ & & M & A & . & . & $\mathrm{C}$ & . & . & . & . & . & . & . & . & F & . & . & . & . & . & . & . & $\mathrm{C}$ & F & . & $\mathbf{R}$ & . & . \\
\hline 20R-CC & & M & A & . & . & $\mathrm{C}$ & . & . & . & . & $\mathbf{R}$ & . & . & . & $\mathrm{F}$ & . & . & . & . & . & . & . & C & $\mathrm{F}$ & . & $\mathbf{R}$ & . & . \\
\hline $21 R-1$ & & & & . & . & . & . & . & . & . & . & . & . & . & . & . & . & . & . & . & . & . & $\cdot$ & . & . & . & . & . \\
\hline $21 \mathrm{R}-2$ & & & & . & . & . & . & . & . & . & . & . & . & . & . & . & . & . & . & . & . & . & . & . & . & . & . & . \\
\hline $21 \mathrm{R}-\mathrm{CC}$ & & & & . & . & . & . & . & . & . & . & ${ }^{\circ}$ & . & : & , & . & . & . & . & . & . & . & . & . & . & . & . & $\dot{0}$ \\
\hline $10 \mathrm{R}-3,13-14$ & & $\mathrm{M}-\mathrm{P}$ & A & . & . & . & . & . & . & . & $\mathrm{F}$ & . & . & $\mathrm{F}$ & . & . & A & F & F & . & . & . & . & . & . & . & C & $\mathrm{F}$ \\
\hline
\end{tabular}


Table 2 (continued).

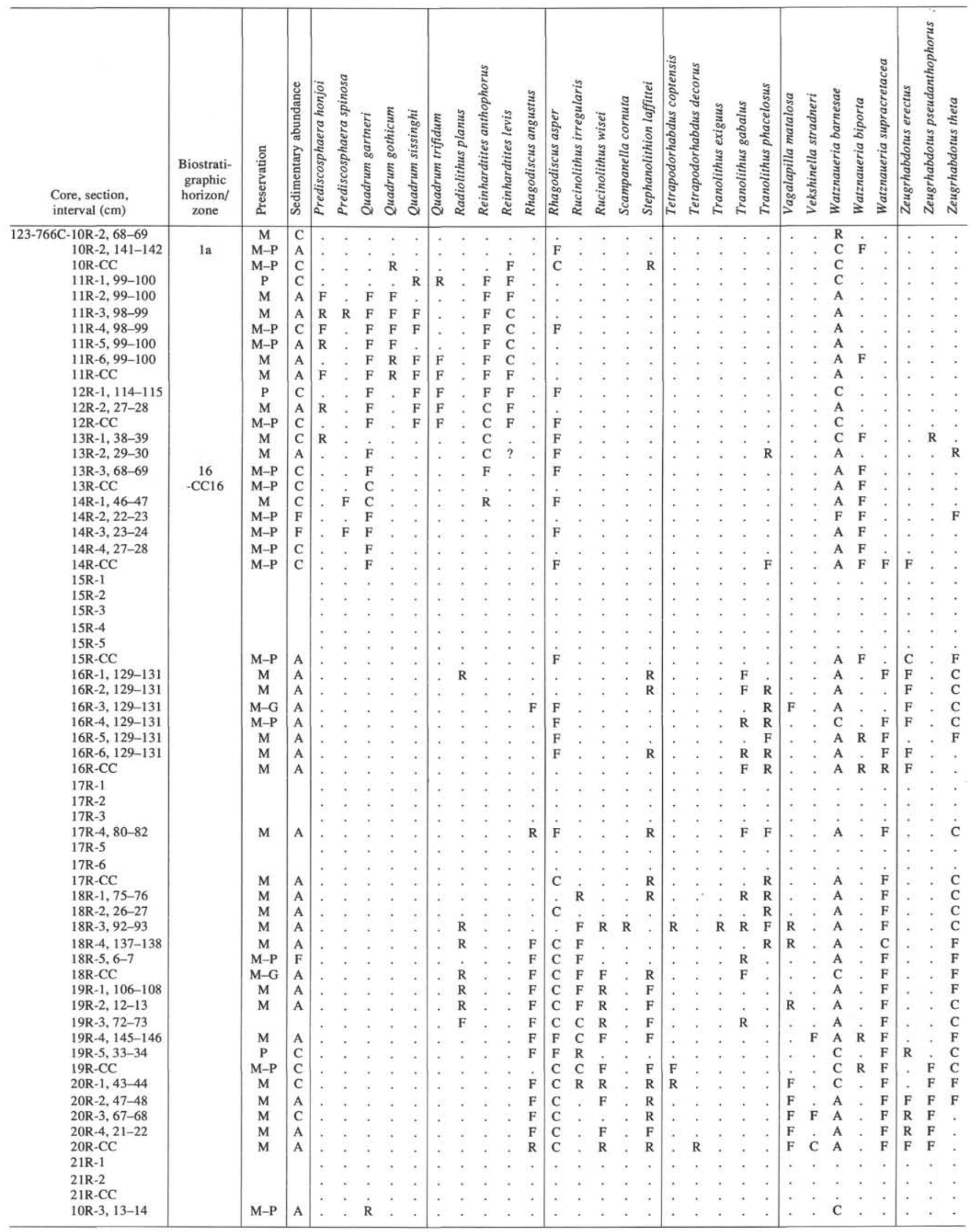


Table 3. Cretaceous calcareous nannofossils from Site 766.

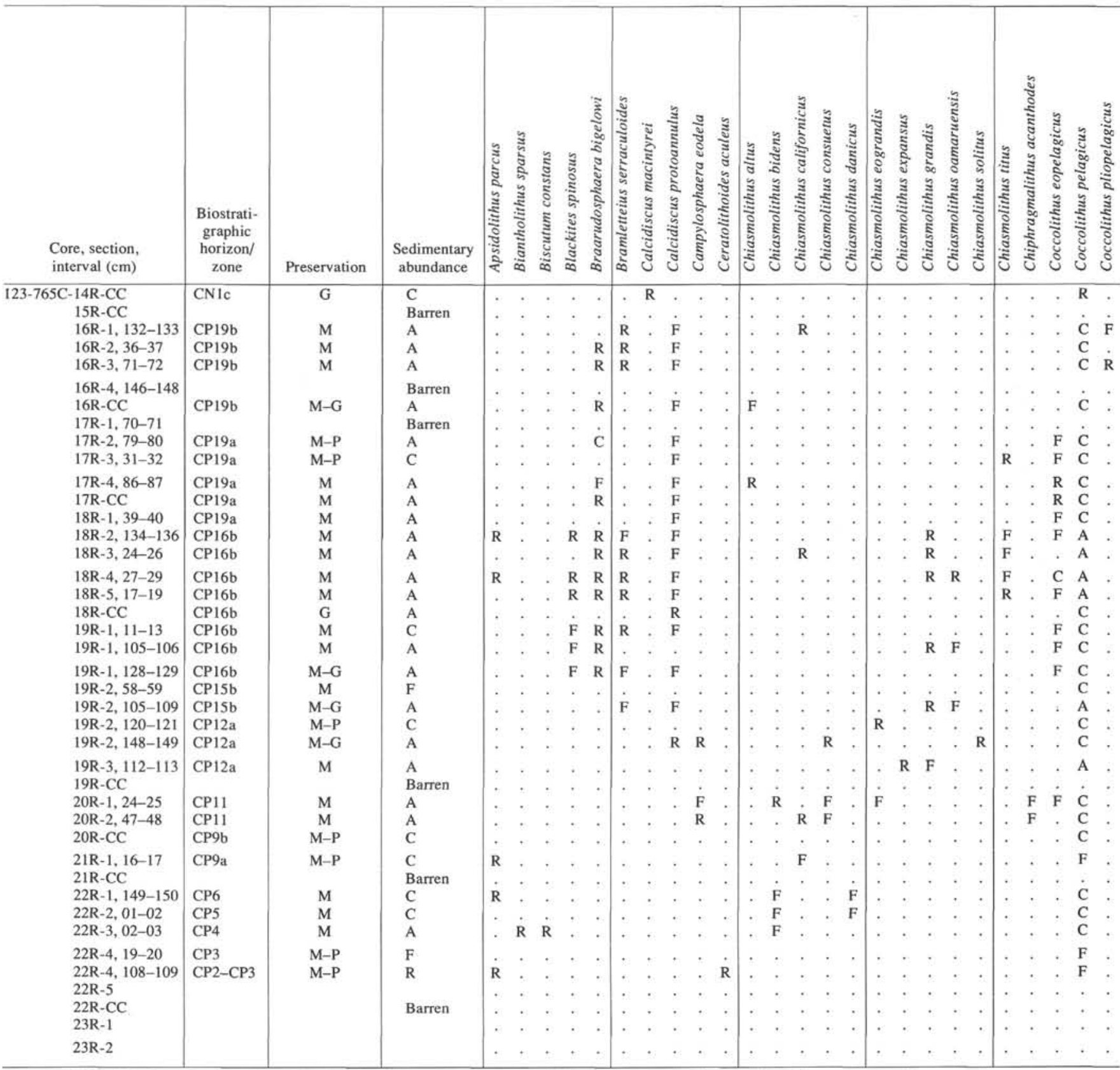

Key to symbols: $\mathrm{RE}=$ Reworked; $\mathrm{R}=$ Rare; $\mathrm{F}=\mathrm{Few} ; \mathrm{C}=$ Common; $\mathrm{A}=\mathrm{Abundant} ; \mathrm{VA}=$ Very abundant; $?=$ Questionable presence; $=$ Not present.

(Calculites obscurus Zone) based on the presence of C. obscurus, Reinhardtites anthophorus, and $M$. furcatus and are late Santonian/early Campanian in age. The stratigraphic gap between Samples 123-765C-25R-2, 48-49 cm, and -25R-1, 33-35 cm, suggests a significant decrease in sedimentation rate (highly condensed section) or the presence of an unconformity or unconformities in this interval. Good recovery in Core $123-765 \mathrm{C}-25 \mathrm{R}$ (71.6\%, Ludden, Gradstein, et al., 1990) supports this interpretation because this suggests that little material was lost because of drilling.

Material between Samples 123-765C-24R-3, 55-58 cm, and 123-765-24R-1, 36-37 cm, was placed in Zone CC19 (Calculites ovalis Zone) based on the presence of Aspidolithus parcus, $R$. anthophorus, $C$. obscurus, and the last appearance of $M$. furcatus in Sample 123-765C-24R-4, 41-43 cm. This zonal assignment indicates an age of middle Campanian. Considering the relative length of Zone CC18 (Aspidolithus parcus Zone), a condensed section or unconformity may exist between Samples 123-765C$24 \mathrm{R}-4,41-43 \mathrm{~cm}$, and $-24 \mathrm{R}-3,55-58 \mathrm{~cm}$ (slightly more than 1.5 $\mathrm{m})$. Fairly good recovery in Core $123-765 \mathrm{C}-24 \mathrm{R}$ (58.9\%, Ludden, Gradstein, et al., 1990)) supports this interpretation. Middle Campanian sediments also were found in Sample 123-765C-23R-CC, which was placed in Zone CC20 (Ceratolithoides aculeus Zone) based on the presence of $C$. aculeus. Samples 123-765C-23R-4, 93-95 cm, and -23R-3, 94-95 cm, were placed in Subzone CC22b (Quadrum trifidum Zone) based on the presence of $Q$. trifidum and are late Campanian in age. The lack of Zone CC21 suggests 
Table 3 (continued).

\begin{tabular}{|c|c|c|c|c|c|c|c|c|c|c|c|c|c|c|c|c|c|c|c|c|c|c|c|c|}
\hline $\begin{array}{l}\text { Core, section, } \\
\text { interval }(\mathrm{cm})\end{array}$ & $\begin{array}{l}\text { Biostrati- } \\
\text { graphic } \\
\text { horizon/ } \\
\text { zone }\end{array}$ & Preservation & $\begin{array}{c}\text { Sedimentary } \\
\text { abundance }\end{array}$ & 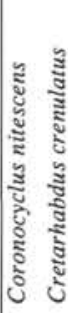 & 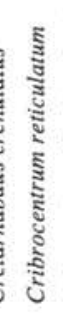 & 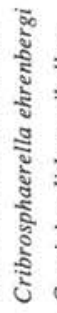 & 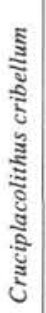 & 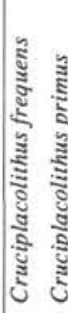 & 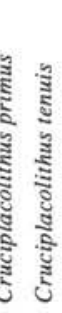 & 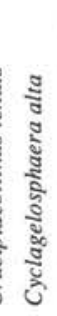 & 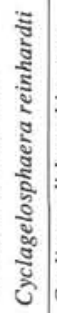 & 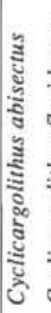 & 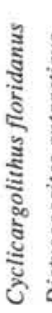 & 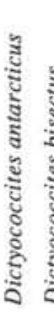 & & 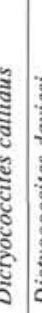 & 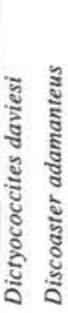 & 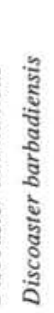 & 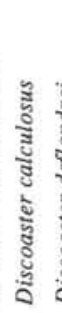 & 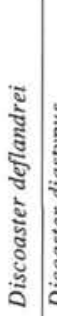 & 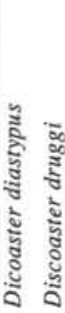 & 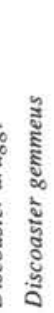 & 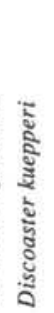 & 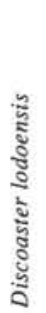 \\
\hline \begin{tabular}{|c|}
$123-765 \mathrm{C}-14 \mathrm{R}-\mathrm{CC}$ \\
$15 \mathrm{R}-\mathrm{CC}$ \\
$16 \mathrm{R}-1,132-133$ \\
$16 \mathrm{R}-2,36-37$ \\
$16 \mathrm{R}-3,71-72$
\end{tabular} & $\begin{array}{l}\text { CN1c } \\
\text { CP19b } \\
\text { CP19b } \\
\text { CP19b }\end{array}$ & $\begin{array}{l}\mathrm{G} \\
\mathrm{M} \\
\mathrm{M} \\
\mathrm{M}\end{array}$ & $\begin{array}{l}\text { C } \\
\text { Barren } \\
\text { A } \\
\text { A } \\
\text { A }\end{array}$ & $\begin{array}{ll}\mathrm{F} & : \\
\dot{\mathrm{F}} & : \\
\mathrm{F} & :\end{array}$ & : & : & $\therefore$ & 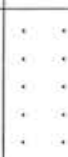 & $::$ & $\therefore$ & $\therefore$ & $\begin{array}{l}\dot{c} \\
\dot{\mathrm{C}} \\
\mathrm{F} \\
\mathrm{F}\end{array}$ & $\begin{array}{l}\text { C } \\
\dot{A} \\
\text { A } \\
\text { A }\end{array}$ & $\begin{array}{l}\mathrm{R} \\
\vdots \\
\vdots\end{array}$ & $\begin{array}{l}\dot{\dot{C}} \\
\dot{\mathrm{C}} \\
\mathrm{C} \\
\mathrm{C}\end{array}$ & : & $\begin{array}{ll}\therefore \\
\therefore \\
\therefore\end{array}$ & $\begin{array}{l}\mathrm{R} \\
\mathrm{R} \\
\mathrm{R}\end{array}$ & $\begin{array}{l}\mathrm{R} \\
\therefore \\
:\end{array}$ & $\begin{array}{l}\mathrm{C} \\
\dot{\mathrm{A}} \\
\mathrm{C} \\
\mathrm{A}\end{array}$ & : & : & $\therefore$ & : \\
\hline $\begin{array}{l}\text { 16R-4, 146-148 } \\
16 \mathrm{R}-\mathrm{CC} \\
17 \mathrm{R}-1,70-71 \\
17 \mathrm{R}-2,79-80 \\
17 \mathrm{R}-3,31-32\end{array}$ & $\begin{array}{l}\text { CP19b } \\
\text { CP19a } \\
\text { CP19a }\end{array}$ & $\begin{array}{l}\mathrm{M}-\mathrm{G} \\
\mathrm{M}-\mathrm{P} \\
\mathrm{M}-\mathrm{P}\end{array}$ & $\begin{array}{l}\text { Barren } \\
\text { A } \\
\text { Barren } \\
\text { A } \\
\text { C }\end{array}$ & $\begin{array}{l}\dot{\mathrm{F}} \\
\dot{\mathrm{F}} \\
\mathrm{R}\end{array}$ & $\dot{\mathrm{R}}$ & $:$ & $\therefore$ & $: \quad:$ & $:$ : & $\therefore$ & $:$ & $\begin{array}{l}\mathrm{F} \\
\dot{\mathrm{C}} \\
\mathrm{C}\end{array}$ & $\begin{array}{l}\dot{\vdots} \\
\dot{A} \\
\mathrm{~A}\end{array}$ & $\dot{\mathrm{v}}$ & $\begin{array}{l}\mathrm{F} \\
\dot{\mathrm{C}} \\
\mathrm{C}\end{array}$ & : & $\begin{array}{ll}\therefore & \dot{R} \\
\therefore & \mathrm{F} \\
& \mathrm{R}\end{array}$ & $\dot{\mathrm{R}}$ & $:$ & $\begin{array}{l}\dot{\mathrm{A}} \\
\dot{\mathrm{A}} \\
\mathrm{A}\end{array}$ & : & : & : & : \\
\hline $\begin{array}{l}17 R-4,86-87 \\
17 R-C C \\
18 R-1,39-40 \\
18 R-2,134-136 \\
18 R-3,24-26\end{array}$ & $\begin{array}{l}\text { CP19a } \\
\text { CP19a } \\
\text { CP19a } \\
\text { CP16b } \\
\text { CP16b }\end{array}$ & $\begin{array}{l}\mathrm{M} \\
\mathrm{M} \\
\mathrm{M} \\
\mathrm{M} \\
\mathrm{M}\end{array}$ & $\begin{array}{l}\mathrm{A} \\
\mathrm{A} \\
\mathrm{A} \\
\mathrm{A} \\
\mathrm{A}\end{array}$ & $\begin{array}{l}\mathrm{F} \\
\dot{\mathrm{F}} \\
\mathrm{F}\end{array}$ & $\begin{array}{l}\mathrm{R} \\
\\
\\
\mathrm{R} \\
\mathrm{R}\end{array}$ & $\dot{\mathrm{R}}$ & $\begin{array}{l}: \\
: \\
:\end{array}$ & $\begin{array}{l}. \\
: \quad: \\
: \quad:\end{array}$ & $:$ & : & $:$ & $\begin{array}{l}\mathrm{F} \\
\mathrm{C} \\
\mathrm{C} \\
\vdots\end{array}$ & $\begin{array}{l}\text { A } \\
\text { A } \\
\text { A } \\
\text { A } \\
\text { A }\end{array}$ & $:$ & $\begin{array}{l}\mathrm{F} \\
\mathrm{F} \\
\text { C } \\
\mathrm{A}\end{array}$ & : & $\begin{array}{ll} & \mathrm{F} \\
\dot{\mathrm{F}} & \mathrm{F} \\
\mathrm{F} & \text { : }\end{array}$ & $\begin{array}{l}\text { : } \\
\text { R } \\
\mathrm{R}\end{array}$ & $:$ & $\begin{array}{l}\mathrm{F} \\
\mathrm{C} \\
\mathrm{C} \\
\mathrm{C}\end{array}$ & : & : & : & : \\
\hline $\begin{array}{l}\text { 18R-4, 27-29 } \\
18 \mathrm{R}-5,17-19 \\
18 \mathrm{R}-\mathrm{CC} \\
\text { 19R-1, 11-13 } \\
\text { 19R-1, 105-106 }\end{array}$ & $\begin{array}{l}\text { CP16b } \\
\text { CP16b } \\
\text { CP16b } \\
\text { CP16b } \\
\text { CP16b }\end{array}$ & $\begin{array}{l}\mathrm{M} \\
\mathrm{M} \\
\mathrm{G} \\
\mathrm{M} \\
\mathrm{M}\end{array}$ & $\begin{array}{l}\text { A } \\
\text { A } \\
\text { A } \\
\text { C } \\
\text { A }\end{array}$ & $\begin{array}{l:}\mathrm{R} \\
\dot{\mathrm{R}} \\
\dot{\mathrm{R}} \\
\mathrm{F}\end{array}$ & $\begin{array}{l}\mathrm{R} \\
: \\
:\end{array}$ & $\begin{array}{l}\dot{\mathrm{R}} \\
\dot{\cdot}\end{array}$ & $\begin{array}{l}: \\
: \\
:\end{array}$ & $\begin{array}{l}: \quad: \\
: \quad: \\
: \quad:\end{array}$ & $:$ & : & $:$ & $:$ & $\begin{array}{l}\text { A } \\
\text { A } \\
\text { A } \\
\text { A } \\
\text { A }\end{array}$ & $:$ & $\begin{array}{l}\mathrm{A} \\
\mathrm{C} \\
\mathrm{C} \\
\mathrm{C} \\
\mathrm{C}\end{array}$ & $\dot{.}$ & $\begin{array}{l}\dot{\mathrm{F}} \\
:\end{array}$ & $\begin{array}{l}\dot{\mathrm{R}} \\
\dot{\mathrm{R}}\end{array}$ & $:$ & $\begin{array}{l}\mathrm{C} \\
\mathrm{F} \\
\dot{\mathrm{F}} \\
\mathrm{C}\end{array}$ & : & : & $\begin{array}{l}\dot{\mathrm{R}} \\
\dot{5} \\
\dot{.}\end{array}$ & : \\
\hline $\begin{array}{l}19 \mathrm{R}-1,128-129 \\
19 \mathrm{R}-2,58-59 \\
19 \mathrm{R}-2,105-109 \\
19 \mathrm{R}-2,120-121 \\
19 \mathrm{R}-2,148-149\end{array}$ & $\begin{array}{l}\text { CP16b } \\
\text { CP15b } \\
\text { CP15b } \\
\text { CP12a } \\
\text { CP12a }\end{array}$ & $\begin{array}{l}M-G \\
M \\
M-G \\
M-P \\
M-G\end{array}$ & $\begin{array}{l}\text { A } \\
\text { F } \\
\text { A } \\
\text { C } \\
\text { A }\end{array}$ & $\begin{array}{l}\mathrm{F} \\
\mathrm{F}\end{array}$ & R & $:$ & $\therefore$ & $\begin{array}{l}: \\
: \\
:\end{array}$ & i. & $\therefore$ & : & : & $\begin{array}{l}\text { A } \\
\text { C } \\
\text { A } \\
\text { C } \\
\text { C }\end{array}$ & : & $\begin{array}{l}\text { C } \\
\text { C } \\
\text { C } \\
\text {. }\end{array}$ & . & : & $\begin{array}{l}\mathrm{R} \\
\mathrm{R} \\
\mathrm{C} \\
\mathrm{C} \\
\mathrm{C}\end{array}$ & : & $\begin{array}{l}\mathrm{C} \\
\mathrm{F} \\
\mathrm{F} \\
\mathrm{C} \\
\mathrm{C}\end{array}$ & : & $\therefore$ & $\begin{array}{l}\dot{\mathrm{C}} \\
\mathrm{F}\end{array}$ & $\begin{array}{l}\dot{ } \\
\dot{\mathrm{C}} \\
\mathrm{C}\end{array}$ \\
\hline $\begin{array}{l}19 \mathrm{R}-3,112-113 \\
19 \mathrm{R}-\mathrm{CC} \\
20 \mathrm{R}-1,24-25 \\
20 \mathrm{R}-2,47-48 \\
\text { 20R-CC }\end{array}$ & $\begin{array}{l}\text { CP12a } \\
\text { CP11 } \\
\text { CP11 } \\
\text { CP9b }\end{array}$ & $\begin{array}{c}\mathrm{M} \\
\mathrm{M} \\
\mathrm{M} \\
\mathrm{M}-\mathrm{P}\end{array}$ & $\begin{array}{l}\text { A } \\
\text { Barren } \\
\text { A } \\
\text { A } \\
\text { C }\end{array}$ & $\dot{:}:$ & : & $:$ & $\begin{array}{l}\mathrm{F} \\
:\end{array}$ & $\begin{array}{l}: \\
\vdots \\
:\end{array}$ & . & F & $\vdots$ & : & F & : & & . & $:$ & $\begin{array}{l}\mathrm{C} \\
\dot{\mathrm{F}} \\
\mathrm{C} \\
\mathrm{C}\end{array}$ & : & $\begin{array}{l}\dot{\mathrm{F}} \\
\mathrm{C}\end{array}$ & $\begin{array}{l}\text { F } \\
\mathrm{F} \\
\mathrm{C}\end{array}$ & $\begin{array}{l}\mathrm{R} \\
\vdots\end{array}$ & $\begin{array}{l}\mathrm{R} \\
\dot{\mathrm{C}} \\
\mathrm{F}\end{array}$ & \\
\hline $\begin{array}{l}21 \mathrm{R}-1,16-17 \\
21 \mathrm{R}-\mathrm{CC} \\
22 \mathrm{R}-1,149-150 \\
22 \mathrm{R}-2,01-02 \\
22 \mathrm{R}-3,02-03\end{array}$ & $\begin{array}{l}\text { CP9a } \\
\text { CP6 } \\
\text { CP5 } \\
\text { CP4 }\end{array}$ & $\begin{array}{l}\mathrm{M} \\
\mathrm{M} \\
\mathrm{M}\end{array}$ & $\begin{array}{l}\text { C } \\
\text { Barren } \\
\text { C } \\
\text { C } \\
\text { A }\end{array}$ & $\begin{array}{ll}: & : \\
: & \mathrm{R} \\
\mathrm{R}\end{array}$ & $:$ & $\dot{\mathrm{R}}$ & $:$ & $\begin{array}{l:}\dot{0} \\
\mathrm{R} \\
\mathrm{R}\end{array}$ & $\begin{array}{ll} & \mathrm{F} \\
\cdot & \dot{\mathrm{F}} \\
\dot{\mathrm{R}} & \mathrm{F} \\
\mathrm{R}\end{array}$ & 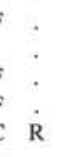 & $\begin{array}{l}\mathrm{R} \\
\dot{\mathrm{R}} \\
\dot{\mathrm{r}}\end{array}$ & : & F & : & & . & : & : & : & $\therefore$ & $\therefore$ & : & : & : \\
\hline $\begin{array}{l}22 \mathrm{R}-4,19-20 \\
22 \mathrm{R}-4,108-109 \\
22 \mathrm{R}-5 \\
22 \mathrm{R}-\mathrm{CC} \\
23 \mathrm{R}-1 \\
23 \mathrm{R}-2\end{array}$ & $\begin{array}{l}\text { CP3 } \\
\text { CP2-CP3 }\end{array}$ & $\begin{array}{l}\text { M-P } \\
\text { M-P }\end{array}$ & $\begin{array}{l}\mathrm{F} \\
\mathrm{R} \\
\text { Barren }\end{array}$ & $\begin{array}{l}\mathrm{R} \\
: \quad: \\
: \\
:\end{array}$ & $\dot{.}$ & $\dot{:}:$ & : & $:$ & $\begin{array}{l}\mathrm{F} \\
\mathrm{R} \\
: \\
:\end{array}$ & : & i. & : & : & & & . & : & : & : & : & : & : & : & \\
\hline
\end{tabular}

another condensed section/unconformity between Samples 123765C-23R-CC and $-23 \mathrm{R}-4,93-95 \mathrm{~cm}$. Good recovery in Core 123-765C-23R(70.6\%, Ludden, Gradstein, et al., 1990) supports this interpretation.

Distinction of Maestrichtian material and the Cretaceous/Tertiary boundary was difficult in sediments from Hole $765 \mathrm{C}$ because of the absence of nannofossils in much of the sampled material and because of restrictions on sampling of critical boundary material (Cores 123-765C-23R and -22R). An age of early Paleogene was found in Sample 123-765C-22R-4, 108-109 cm, which was placed in undifferentiated Zones CP2-CP3 (Chiasmolithus danicus-Ellipsolithus macellus Zones) based on the presence of Cruciplacolithus tenuis and Toweius eminens (Fig. 3). Extensive reworking of Late Cretaceous nannofossils occurs in this sample. Early Paleocene material also can be found in Sample 123-765C-
$22 \mathrm{R}-4,19-20 \mathrm{~cm}$, which was tentatively placed in Zone CP3 and in Sample 123-765C-22R-3, 2-3 cm, which was placed in Zone CP4 (Fasciculithus tympaniformis Zone) based on the presence of $F$. tympaniformis. Late Paleocene material was found in Samples 123-765C-22R-2, 1-2 cm, and 123-765C-22R-1, 149-150 $\mathrm{cm}$, which were placed in Zones CP5 (Heliolithus kleinpelli Zone) and CP6 (Discoaster mohleri Zone), respectively, based on the presence of both $H$. kleinpelli and D. mohleri.

Eocene sediment first occurs in Sample 123-765C-21R-1, 16$17 \mathrm{~cm}$, and was placed in Subzone CP9a (Tribrachiatus contortus Subzone) based on the presence of Discoaster diastypus. Poor recovery in Core $23-765 \mathrm{C}-21 \mathrm{R}-1$ (6.8\%, Ludden, Gradstein, et al., 1990) and an intervening barren sample (Sample 123-765C21R-CC) suggest that Zones CC7 (Discoaster nobilis Zone) and CC8 (Discoaster multiradiatus Zone) may be present between 
Table 3 (continued).

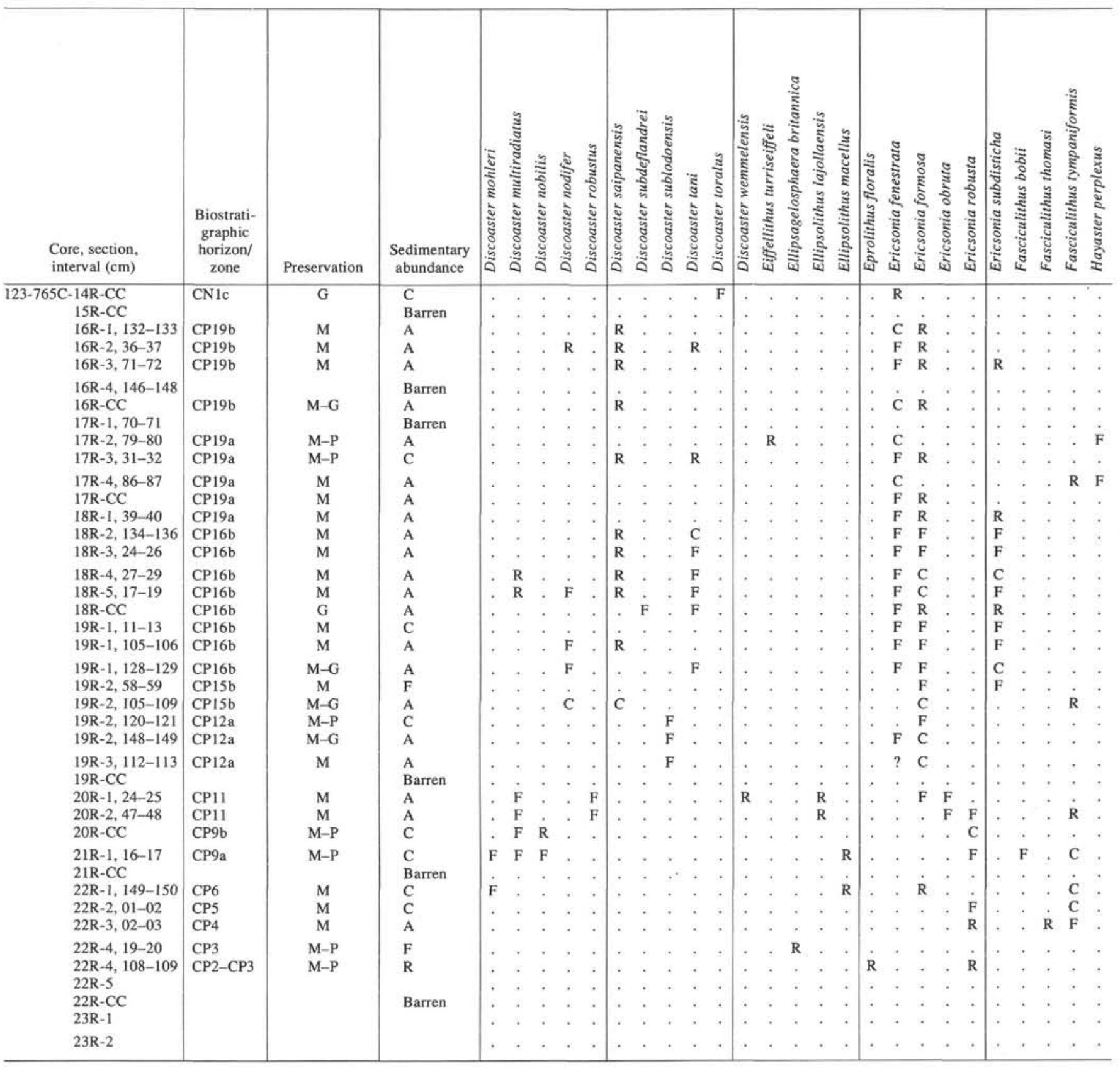

Samples $123-765 C-22 \mathrm{R}-1,149-150 \mathrm{~cm}$, and $-21 \mathrm{R}-1,16-17 \mathrm{~cm}$. However, the presence of a condensed section/unconformity in this interval cannot be ruled out.

The presence of Tribrachiatus orthostylus and last appearance of $F$. tympaniformis indicates Subzone CP9b (Discoaster binodosus Subzone) and an age of early Eocene for Sample 123-765C20R-CC. Samples 123-765C-20R-2, 47-48 cm, and 20R-1, 24-25 $\mathrm{cm}$, were placed in Zone CP11 (Discoaster lodoensis Zone) based on the presence of $D$. lodoensis and are early Eocene in age. Poor recovery in Core $123-765 \mathrm{C}-20 \mathrm{R}$ (25.9\%, Ludden, Gradstein, et al., 1990) may be responsible for the absence of Zone CP10.

Sediments between Samples 123-765C-19R-3, 112-113 cm, through $-19 \mathrm{R}-2,120-121 \mathrm{~cm}$, were placed in Subzone CP12a (Discoasteroides kuepperi Subzone) based on the presence of Discoaster sublodoensis and are early Eocene in age. Samples
123-765C-19R-2, 105-109 cm, and -19R-2, 58-59 cm, were placed in Subzone CP15b (Isthmolithus recurvus Subzone) based on the presence of $I$. recurvus. A significant unconformity exists between Samples 123-765C-19R-2, 120-121 cm, and 123-765C$19 \mathrm{R}, 105-109 \mathrm{~cm}$, as indicated by the absence of most of the late Eocene (Zones CP13 and CP14 and Subzone CP15a). Fairly good recovery in Core $123-765 \mathrm{C}-19 \mathrm{R}$ (47.9\%, Ludden, Gradstein, et al., 1990) supports this interpretation. According to Berggren et al. (1985) this hiatus should span at least $12 \mathrm{Ma}$.

Early Oligocene sediments were first found in Sample 123765C-19R-1, 128-129 cm, and continues through Sample 123$765 \mathrm{C}-18 \mathrm{R}-2,134-136 \mathrm{~cm}$. This interval was placed in Subzone CP16b (Ericsonia formosa Subzone) based on the presence of $E$. formosa and $I$. recurvus. The next available sample (Sample 123-765C-18R-1, 39-40 cm) was placed in Subzone CP19a $(C y$ - 
Table 3 (continued).

\begin{tabular}{|c|c|c|c|c|c|c|c|c|c|c|c|c|c|c|c|c|c|c|c|c|c|c|c|c|c|c|}
\hline $\begin{array}{l}\text { Core, section, } \\
\text { interval }(\mathrm{cm})\end{array}$ & $\begin{array}{l}\text { Biostrati- } \\
\text { graphic } \\
\text { horizon/ } \\
\text { zone }\end{array}$ & Preservation & $\begin{array}{c}\text { Sedimentary } \\
\text { abundance }\end{array}$ & 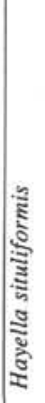 & 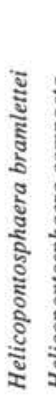 & 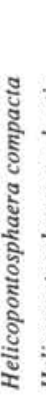 & 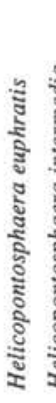 & 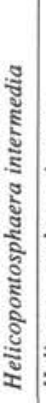 & 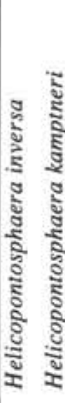 & 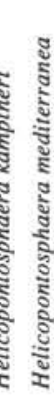 & 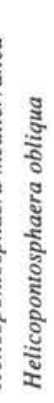 & 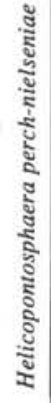 & 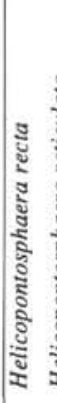 & 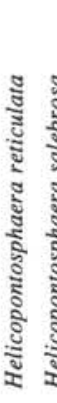 & 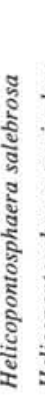 & 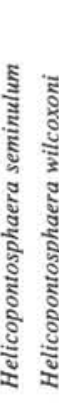 & & 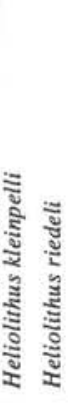 & 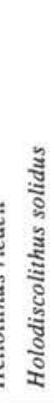 & 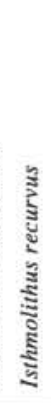 & 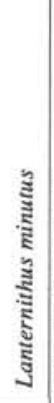 & 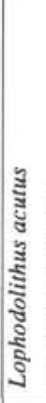 & 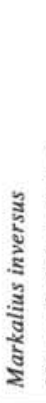 & 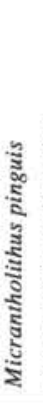 & 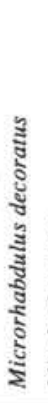 & \\
\hline $123-765 \mathrm{C}-14 \mathrm{R}-\mathrm{CC}$ & CNIc & G & C & . & . & . & $\mathrm{F}$ & & . $\mathrm{R}$ & $\mathbf{R}$. & . & $\cdot$ & . & . & . & . & & . & . & . & $\cdot$ & . & $\cdot$ & . & . & . \\
\hline 15R-CC & & & Barren & . & . & $\therefore$ & $\dot{5}$ & & . & . & & . & $\dot{r}$ & . & . & . & • & . & - & $\dot{\mathrm{D}}$ & $\dot{p}$ & . & . & . & . & . \\
\hline $16 \mathrm{R}-1,132-133$ & CP19b & M & A & & . & $\mathbf{R}$ & $\mathrm{F}$ & & . & . & $\mathbf{R}$ & . & $\mathrm{F}$ & . & . & . & . & . & . & $\mathbf{R}$ & $\mathbf{R}$ & . & . & . & . & . \\
\hline $16 \mathrm{R}-2,36-37$ & CP19b & M & A & $\mathrm{R}$ & . & $\mathbf{R}$ & $\mathrm{F}$ & & . & . & $\mathrm{R}$ & . & $\mathrm{F}$ & $\mathbf{R}$ & . & . & . & . & . & . & $\mathbf{R}$ & . & . & . & . & . \\
\hline $16 \mathrm{R}-3,71-72$ & CP19b & M & A & $\mathbf{R}$ & . & $\mathbf{R}$ & $\mathrm{F}$ & & . & $\mathbf{R}$ & . & . & $\mathrm{F}$ & . & . & . & . & . & - & - & $\mathbf{R}$ & - & . & - & . & . \\
\hline $\begin{array}{l}16 \mathrm{R}-4,146-148 \\
16 \mathrm{R}-\mathrm{CC}\end{array}$ & CP19b & & $\begin{array}{l}\text { Barren } \\
\text { A }\end{array}$ & . & . & : & $\dot{\mathrm{F}}$ & & . & . & $\dot{\mathrm{F}}$ & · & & $\dot{.}$ & . & : & . & . & . & : & · & · & · & . & · & . \\
\hline $\begin{array}{l}10 \mathrm{R}-\mathrm{CC} \\
17 \mathrm{R}-1,70-71\end{array}$ & СP19b & $M-G$ & $\begin{array}{l}\text { A } \\
\text { Barren }\end{array}$ & : & $\dot{.}$ & $\dot{.}$ & F & & : & . $\mathrm{R}$ & $\mathrm{F}$ & $\therefore$ & $\mathrm{C}$ & $\dot{.}$ & & $\therefore$ & ? & . & : & $\dot{.}$ & 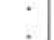 & $\dot{.}$ & $\dot{0}$ & : & $\therefore$ & : \\
\hline $17 \mathrm{R}-2,79-80$ & CP19a & M-P & A & . & : & . & $\dot{\mathrm{F}}$ & & : & : & $\dot{\mathrm{F}}$ & ; & $\dot{\mathrm{C}}$ & : & : & . & : & : & $\mathrm{F}$ & . & . & . & . & R & . & : \\
\hline $17 \mathrm{R}-3,31-32$ & CP19a & M-P & C & . & . & , & . & & . & . & . & . & $\mathrm{F}$ & . & . & & . & & . & . & $\mathbf{R}$ & . & . & . & . & . \\
\hline $17 \mathrm{R}-4,86-87$ & CP19a & $\mathrm{M}$ & A & R & . & $\dot{.}$ & $\mathrm{F}$ & & . & . & $F$ & . & C & . & . & . & . & . & . & . & $\mathbf{R}$ & . & . & R & . & . \\
\hline 17R-CC & CP19a & M & A & . & . & $\mathrm{R}$ & $\mathrm{F}$ & & . & . & . & . & $\mathrm{F}$ & . & . & . & & . & . & . & . & . & . & . & . & . \\
\hline $18 \mathrm{R}-1,39-40$ & CP19a & M & A & . & . & . & F & & . & . & $\mathbf{R}$ & . & $\mathrm{F}$ & . & . & . & & . & . & . & $\mathrm{F}$ & . & . & . & . & . \\
\hline $18 \mathrm{R}-2,134-136$ & CP16b & M & A & & & $\mathrm{F}$ & . & & . & . & . & . & $\mathrm{F}$ & F & . & . & . & & . & $\mathbf{R}$ & & . & . & . & . & . \\
\hline $18 R-3,24-26$ & CP16b & M & A & R & . & C & . & . & . & . & $\mathrm{R}$ & . & $\mathrm{F}$ & $\mathrm{F}$ & . & . & & . & . & R & $\mathrm{F}$ & . & . & . & . & . \\
\hline $18 \mathrm{R}-4,27-29$ & CP16b & M & A & F & . & $\mathrm{F}$ & $\mathrm{R}$ & & . & . & $R$ & F & $\mathbf{R}$ & $\mathrm{F}$ & . & . & & . & . & $\mathbf{R}$ & $\mathrm{C}$ & . & . & . & . & . \\
\hline $18 \mathrm{R}-5,17-19$ & CP16b & M & A & & . & F & $\mathrm{R}$ & & . & . & . & $\mathbf{R}$ & $\mathbf{R}$ & $\mathrm{F}$ & . & . & . & , & . & . & C & . & . & . & . & $\mathbf{R}$ \\
\hline 18R-CC & CP16b & G & A & $\mathbf{R}$ & F & $\mathrm{F}$ & F & & . & . & . & . & $\mathrm{R}$ & C $\mathrm{F}$ & F & . & & . & . & . & C & . & . & . & . & . \\
\hline $19 \mathrm{R}-1,11-13$ & CP16b & M & C & $\mathrm{R}$ & . & $\mathrm{F}$ & F & & . & . & . & . & . & $\mathrm{F}$ & . & . & $\mathrm{F}$ & . & . & . & c & . & . & . & . & . \\
\hline $19 \mathrm{R}-1,105-106$ & CP16b & M & A & . & $\mathrm{F}$ & $\mathrm{F}$ & . 1 & & . & . & . & . & $\mathrm{F}$ & F & . & R & & & A & . & $\mathrm{F}$ & . & . & . & . & . \\
\hline $19 \mathrm{R}-1,128-129$ & CP16b & $M-G$ & A & $\mathrm{F}$ & $\mathrm{F}$ & C & $\mathbf{R}$ & $\mathrm{F}$ & F & . & . & . & F & F & . & $\mathrm{F}$ & $\mathrm{F}$ & . & A & $\mathrm{R}$ & $\mathrm{C}$ & . & . & . & . & . \\
\hline $19 \mathrm{R}-2,58-59$ & CP15b & M & $\mathrm{F}$ & & . & $\mathrm{F}$ & . & . & . & . & . & . & . & R & . & . & & . & . & $\mathbf{R}$ & . & . & . & . & . & . \\
\hline $19 \mathrm{R}-2,105-109$ & CP15b & $M-G$ & A & $\mathbf{R}$ & . & $\mathrm{F}$ & . & . & . & . & . & . & . & $\mathrm{R}$. & . & . & & . & . & F & . & . & . & . & . & . \\
\hline $19 \mathrm{R}-2,120-121$ & CP12a & M-P & c & . & . & . & . & & . & , & , & . & . & . & . & . & & & . & . & . & . & . & . & . & . \\
\hline $19 \mathrm{R}-2,148-149$ & CP12a & $M-G$ & A & . & . & . & . & & . & . & . & . & . & . & . & . & & . & . & . & . & . & . & . & . & . \\
\hline 19R-3, 112-113 & CP12a & M & A & . & . & . & . & $\cdot$ & . & . & . & . & . & . & . & . & & . & . & . & . & R & $?$ & . & . & . \\
\hline 19R-CC & & & Barren & . & . & . & . & & . & . & . & . & . & & & & & & . & . & . & . & . & . & . & - \\
\hline $20 \mathrm{R}-1,24-25$ & CP11 & M & A & . & . & . & . & & . & . & . & . & . & . & . & . & . & . & . & . & . & . & . & . & . & . \\
\hline $20 \mathrm{R}-2,47-48$ & CP11 & M & A & . & . & . & . & . & . & . & . & . & . & . & . & . & & . & . & . & . & . & $\mathbf{R}$ & . & . & \\
\hline 20R-CC & $\mathrm{CP} 9 \mathrm{~b}$ & M-P & C & . & . & . & . & & . & . & . & . & . & . & . & . & & . & . & . & . & . & $\mathrm{F}$ & . & . & R \\
\hline 21R-1, 16-17 & CP9a & $M-P$ & C & , & . & . & . & & . & . & . & . & . & . & . & . & . & $\mathbf{R}$ & . & . & . & . & $\mathbf{R}$ & . & . & . \\
\hline 21R-CC & & & Barren & . & . & . & . & & . & . . & . & . & . & . & . & . & . & . & . & . & . & . & . & . & . & \\
\hline $22 \mathrm{R}-1,149-150$ & CP6 & M & C & . & & & . & & & & & . & & & & & . & c & . & . & . & . & . & . & . & $\mathrm{R}$ \\
\hline $22 \mathrm{R}-2,01-02$ & CP5 & M & C & . & . & . & . & . & . & . & . & . & . & . & . & . & & C & . & . & . & . & . & . & . & $\mathbf{R}$ \\
\hline $22 \mathrm{R}-3,02-03$ & CP4 & $\mathrm{M}$ & A & . & . & $\cdot$ & . & & . & . & . & . & . & . & . & . & & . & . & . & . & . & . & . & . & \\
\hline $22 \mathrm{R}-4,19-20$ & $\mathrm{CP} 3$ & M-P & $\mathrm{F}$ & . & . & . & . & . & . & . & . & . & . & . & . & & & . & . & . & & . & . & , & . & $\mathbf{R}$ \\
\hline $22 \mathrm{R}-4,108-109$ & CP2-CP3 & M-P & $\mathbf{R}$ & . & . & . & . & & . & . & . & . & . & . & . & & & . & . & . & . & . & F & . & $\mathrm{R}$ & $\mathbf{R}$ \\
\hline $22 \mathrm{R}-5$ & & & & . & . & . & . & & . & . & . & . & . & . & . & . & & . & . & . & . & . & . & . & . & . \\
\hline 22R-CC & & & Barren & . & . & . & . & & . & . & . & . & . & . & . & & & . & . & . & . & . & . & . & . & . \\
\hline 23R-1 & & & & . & . & . & . & & & . & . & . & . & . & . & . & & . & . & . & . & . & . & . & . & . \\
\hline $23 \mathrm{R}-2$ & & & & . & . & . & . & & . & . & . & . & . & . & . & & & & . & . & . & . & . & . & . & . \\
\hline
\end{tabular}

clicargolithus floridanus Subzone) based on the presence of Sphenolithus ciperoensis and is late Oligocene in age. The zonal assignment of this sample reveals another significant hiatus comprising most of the early Oligocene, as evidenced by the absence of Zones CP17 and CP18. This is supported by the good recovery of Core 123-765C-18R (78.3\%, Ludden, Gradstein, et al., 1990), which suggests that few sediments were lost because of drilling. According to Berggren et al. (1985), this hiatus should span at least 8.5 Ma. Samples 123-765C-17R-CC through -17R-2, 79-80 $\mathrm{cm}$, also were placed in Subzone CP19a. Samples 123-765C$17 \mathrm{R}-1,70-71 \mathrm{~cm}$, through $-16 \mathrm{R}-\mathrm{CC}$ were placed in Subzone CP19b (Dictyococcites bisectus Subzone) based on the presence of $S$. ciperoensis and last appearance of Sphenolithus distentus in Sample $123-765 \mathrm{C}-17 \mathrm{R}-2,79-80 \mathrm{~cm}$, and also are late Oligocene in age.
The Oligocene/Miocene boundary is indistinguishable in material from Hole $765 \mathrm{C}$ because of the absence of nannofossils in Core 123-765C-15R. The next available sample (Sample 123765C-14R-CC) was placed in Subzone CN1c (Discoaster druggii Subzone) based on the presence of $D$. druggii and is early Miocene in age.

\section{Site 766}

Shipboard sedimentologic work indicated that the Albianlower Eocene section of Hole 766A (Units II through IV) consists primarily of hemipelagic to pelagic zeolitic calcareous nannofossil oozes and clay (Ludden, Gradstein, et al., 1990). Consequently, reworking is minimal, and nannofossils from this interval are generally abundant as a sedimentary component, while assemblages are diverse and moderately to well-preserved (Tables 3 and 
Table 3 (continued).

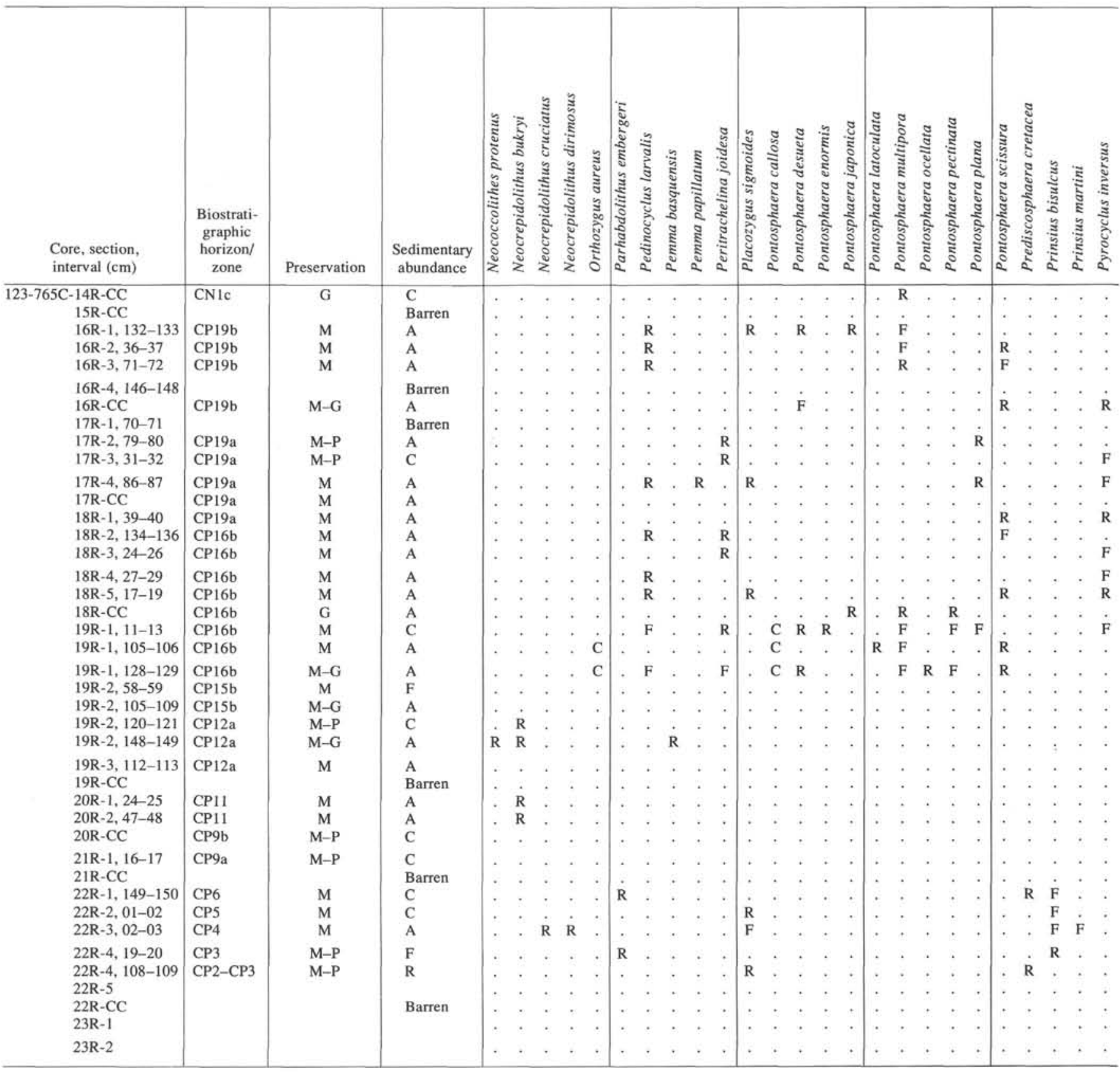

4). Average recovery for the entire sediment section was $63.1 \%$, with a slightly lower average value for the Upper Cretaceous-Paleogene interval (Ludden, Gradstein, et al., 1990). Shipboard biostratigraphic work indicated a nearly complete Albian-early Eocene record (Ludden, Gradstein, et al., 1990), which is confirmed here. Good recovery combined with a relatively complete stratigraphic record and diverse, well-preserved nannofossil assemblages allowed for an accurate, high-resolution biostratigraphic correlation and the establishment of a useful temporal framework for the hole.

At the base of the studied interval, a thick section of Zone CC8 (Prediscosphaera columnata Zone) is present that includes Samples 123-766A-21R-CC through -17R-CC (Fig. 2). The zonal assignment is based on the presence of $P$. columnata and denotes an Albian age. The next available sample (Sample 123-766A-
$17 \mathrm{R}-4,80-82 \mathrm{~cm})$ contains E. turriseiffeli and was placed in Zone CC9 (Eiffellithus turriseiffeli Zone), indicating an age of Albian-Cenomanian. Albian-Cenomanian sediments continue through Sample 123-766A-15R-CC, and all intervening samples also were placed in Zone CC9.

Samples 123-766A-14R-CC and -14R-4, 27-28 cm, were placed in Zone CC11 (Quadrum gartneri Zone), as determined by the presence of $Q$. gartneri, and indicates an age of Turonian. Distinction of Zone CC10 and the Cenomanian/Turonian boundary is impossible here because of sampling restrictions. Samples 123-766A-14R-3, 23-24 cm, and -14R-2, 22-23 cm, were placed in Zones CC12 (Lucianorhabdus maleformis Zone) and CC13 (Marthasterites furcatus Zone), respectively, based on the presence of both $E$. eximius and $M$. furcatus. This denotes an age of late Turonian-Coniacian for these two samples. 
Table 3 (continued).

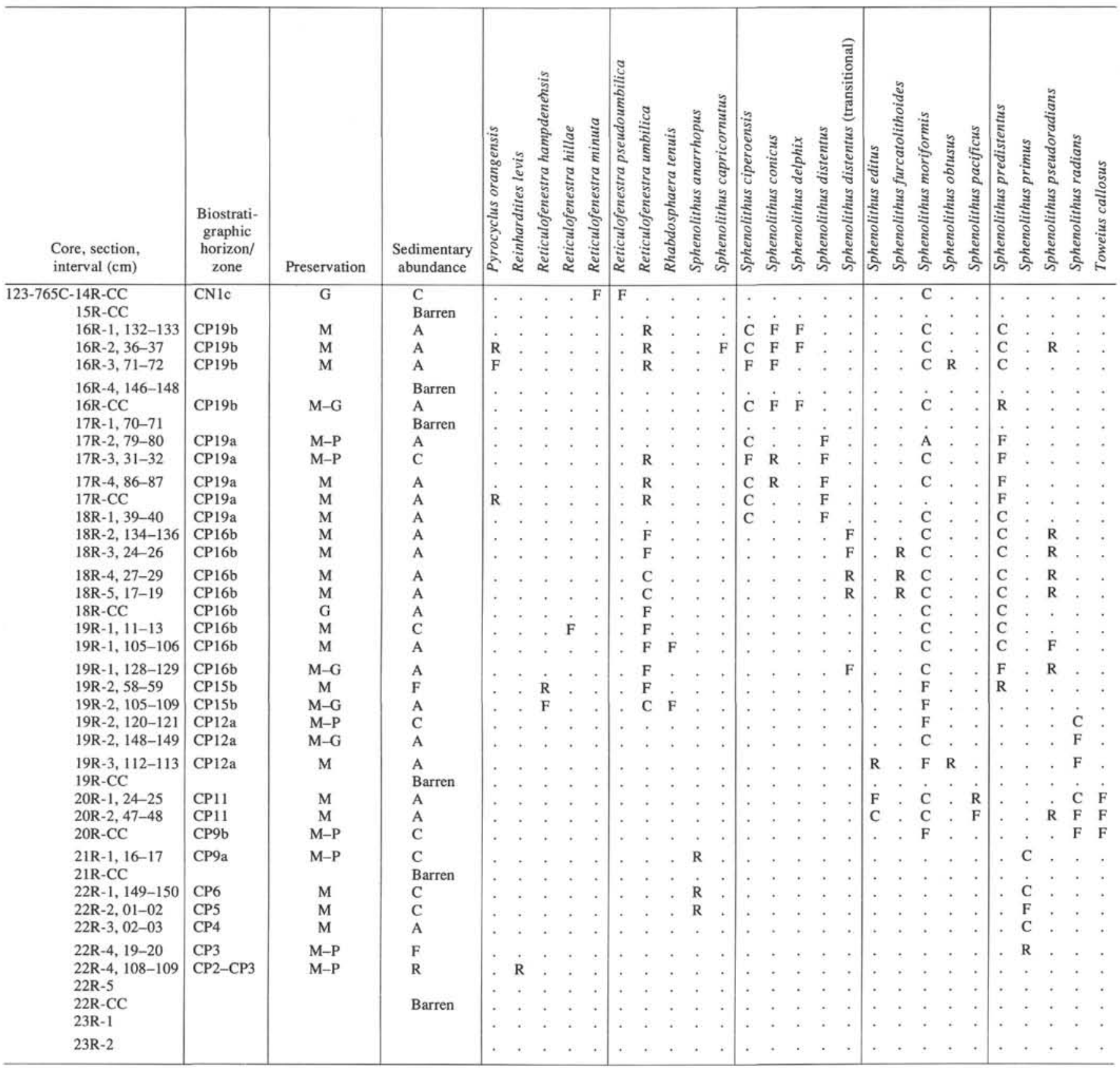

Sample 123-766A-14R-1, 46-47 cm, was placed in Zone $\mathrm{CC} 15$ (Reinhardtites anthophorus Zone) based on the presence of $R$. anthophorus and is of Santonian age. Good recovery in Core 123-766A-14R (>50\%, Ludden, Gradstein, et al., 1990) and the absence of Zone $\mathrm{CC} 14$ suggest an unconformity or condensed section between Samples 123-766A-14R-2, 22-23 cm, and -14R-1, 46-47 cm. Samples 123-766A-13R-CC and 123-766A-13R-3, $68-69 \mathrm{~cm}$, were placed in undifferentiated Zones CC15-CC16 (Reinhardtites anthophorus-Lucianorhabdus cayeuxii zones) based on the presence of species indicative of this zonal interval and also are of Santonian age. The next available samples (Samples 123-766A-13R-2, 29-30 cm, and -13R-1, 38-39 cm) were placed in Zone CC19 (Calculites ovalis Zone) based on the presence of A. parcus and Lucianorhabdus cayeuxii and are early
Campanian in age. Poor recovery in Core 123-766A-13R $(<50 \%$, Ludden, Gradstein, et al., 1990) may account for the absence of Zones CC17 and CC18 between Samples 123-766A-13R-3, 68$69 \mathrm{~cm}$, and $-13 \mathrm{R}-2,29-30 \mathrm{~cm}$; however, the presence of an unconformity or condensed section cannot be ruled out.

Sample 123-766A-12R-CC was placed in Zone CC21 (Quadrum nitidum Zone) based on the presence of $Q$. nitidum and is late Campanian in age. Poor recovery in Core $123-766 \mathrm{~A}-13 \mathrm{R}(<50 \%$, Ludden, Gradstein, et al., 1990) may account for the absence of Zone CC20; however, a disconformity may exist between Samples 123-766A-13R-1, 38-39 cm, and -12R-CC. Samples 123$766 \mathrm{~A}-12 \mathrm{R}-2,27-28 \mathrm{~cm}$, and $-12 \mathrm{R}-1,114-115 \mathrm{~cm}$, were placed in Subzone CC22a (Quadrum trifidum Zone) based on the presence of $Q$. trifidum and are late Campanian in age. Samples 123- 
Table 3 (continued).

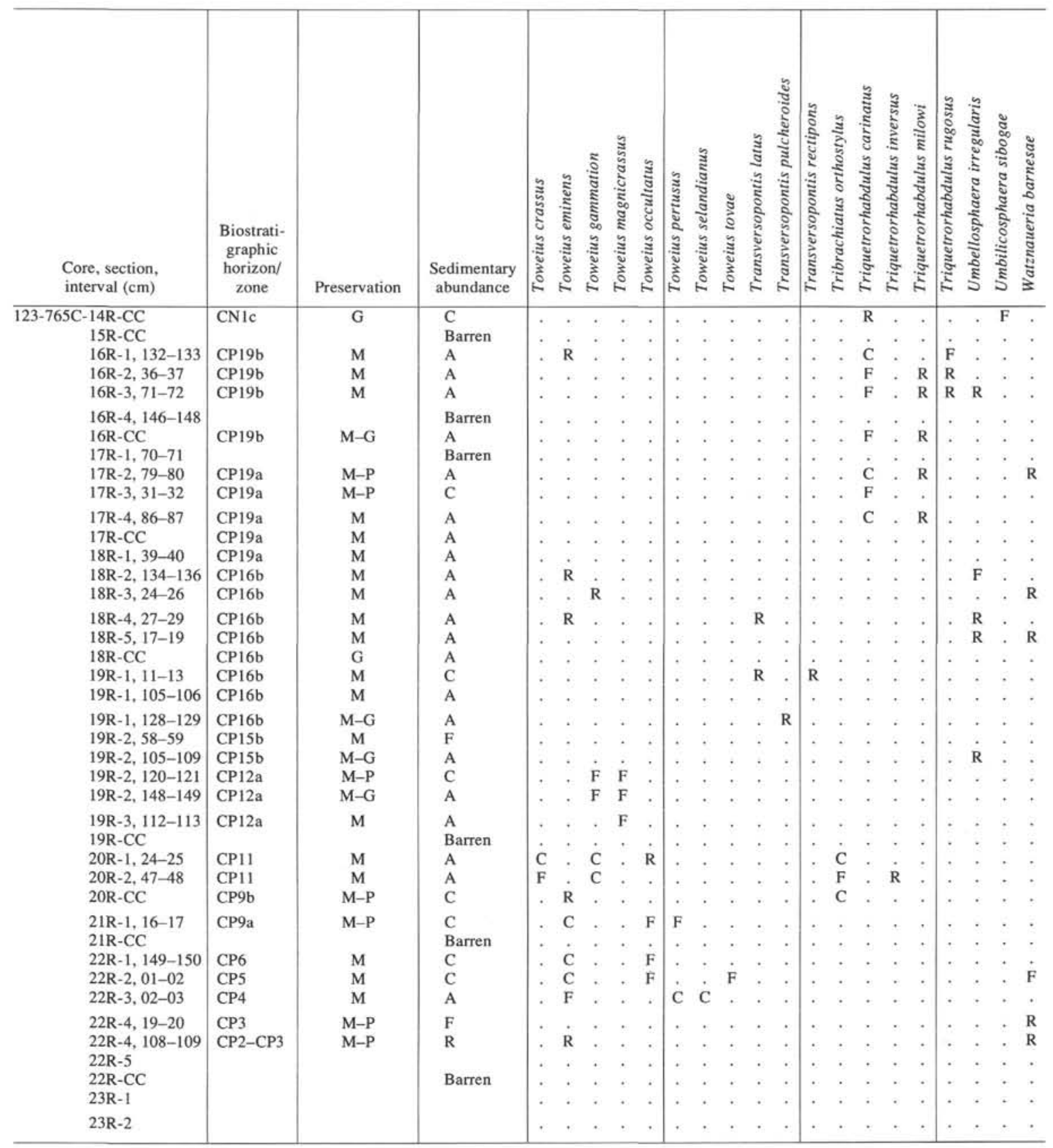

766A11R-CC through -11R-1, 99-100 cm, were placed in Subzone CC22b (Quadrum trifidum Zone) based on the presence of Reinhardtites levis and $Q$. trifidum and are late Campanian in age.

Sample 123-766A-10R-CC was placed in Subzone CP23a (Tranolithus phacelosus Zone) based on the last appearance of $R$. anthophorus and is late Campanian-Maestrichtian in age. The next overlying sample (Sample 123-766A-10R-3, 13-14 cm) was placed in Zone CC26 (Nephrolithus frequens Zone) based on the presence of Micula murus and is late Maestrichtian in age. Poor recovery in Core $123-766 \mathrm{~A}-10 \mathrm{R}(<50 \%$, Ludden, Gradstein, et al., 1990) may be responsible for the lack of significant material of Maestrichtian age, but the presence of an unconformity or condensed section cannot be ruled out.

Cretaceous/Tertiary boundary material was found in Sample 123-766A-10R-2, 141-142 cm, and this sample was placed in the undifferentiated Zone/Subzone CC26-CP1a (Nephrolithus frequens Zone/Cruciplacolithus primus Subzone) based on the presence of Biantholithus sparsus and substantial late Maestrichtian and other reworked Late Cretaceous species. Shipboard sedimentologists found material in Core 123-766A-10R that consisted of a mottled nannofossil ooze, suggesting extensive bioturbation (Ludden, Gradstein, et al., 1990). Reworking and co-occurrence of chronostratigraphically exclusive taxa further support this interpretation. Sample 123-766A-10R-2, 68-69 cm, was confidently placed in Subzone CP1a (Cruciplacolithus primus Subzone) based on the presence of Cruciplacolithus edwardsii and represents an age of early Paleocene.

Samples 123-766A-10R-1, 120-121 cm, through -9R-CC were placed in Subzone CP1b (Cruciplacolithus tenuis Subzone) based on the presence of Cruciplacolithus tenuis s.s. (Hay and Mohler, 


\section{Argo Abyssal Exmouth Plateau \\ Hole $765 \mathrm{C}$ \\ Hole 766A}

\begin{tabular}{|c|c|c|c|c|c|}
\hline \multirow{5}{*}{ 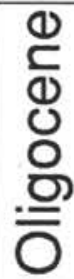 } & \multirow{2}{*}{ S. ciperoensis } & \multirow{2}{*}{ CP19 } & & $16 \mathrm{R} \rightarrow 18 \mathrm{R}-1,40$ & \\
\hline & & & & & \\
\hline & $S$. distentus & CP18 & & & \\
\hline & S. predistentus & CP17 & & & \\
\hline & H. reticulata & CP16 & $\frac{c}{b}$ & $18 R-2,136 \rightarrow 19 R-1,129$ & \\
\hline \multirow{9}{*}{ 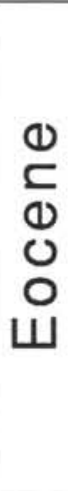 } & D. barbadiensis & \multicolumn{2}{|c|}{$\mathrm{CP}_{15} \frac{\mathrm{b}}{\mathrm{a}}$} & $19 R-2,58 \rightarrow 19 R-2,109$ & \\
\hline & R. umbilica & CP14 & $\frac{b}{a}$ & & \\
\hline & N. quadrata & $\mathrm{CP} 13$ & $\frac{c}{b}$ & & \\
\hline & \multirow{2}{*}{ D. sublodoensis } & \multirow{2}{*}{ CP12 } & b & & \\
\hline & & & a & $19 \mathrm{R}-2,121 \rightarrow 19 \mathrm{R} 3,113$ & $3 R-1,119 \rightarrow 3 R-5,93$ \\
\hline & D. lodoensis & CP11 & & $20 \mathrm{R}-1,26 \rightarrow 20 \mathrm{R}-2,47$ & $3 R-6,116 \rightarrow 4 R-2,102$ \\
\hline & T. orthostylus & CP10 & & & \\
\hline & \multirow{2}{*}{ D. diastypus } & \multirow{2}{*}{ CP9 } & b & 20R-CC & \multirow{2}{*}{$4 \mathrm{R}, \mathrm{CC} \rightarrow 6 \mathrm{R}-5,105$} \\
\hline & & & a & $21 \mathrm{R}-1,17$ & \\
\hline \multirow{7}{*}{$\begin{array}{l}\text { (1) } \\
\text { ᄃ } \\
\text { (1) } \\
0 \\
0 \\
0\end{array}$} & \multirow{2}{*}{ D. multiradiatus } & \multirow{2}{*}{\multicolumn{2}{|c|}{ CP8 $\frac{b}{a}$}} & & $6 \mathrm{R}-6,114 \rightarrow 6 \mathrm{R}-\mathrm{CC}$ \\
\hline & & & & & $7 R$ \\
\hline & D. nobilis & CP7 & & & $8 \mathrm{R}-1,95$ \\
\hline & D. mohlerii & CP6 & & $22 R-1,150$ & $8 \mathrm{R}-2,31 \rightarrow 8 \mathrm{R}-\mathrm{CC}$ \\
\hline & H. kleinpellii & CP5 & & 22R-2, 2 & \\
\hline & F. tympaniformis & $\mathrm{CP} 4$ & & $22 R-3,3$ & \\
\hline & E. macellus & CP3 & & \multirow[b]{2}{*}{$22 \mathrm{R}-4,19-108$} & \multirow[b]{2}{*}{$\begin{array}{l}9 R-1,111 \rightarrow 9 R-3,111 \\
9 R-4,111 \rightarrow 9 R-5,111\end{array}$} \\
\hline a & C. danicus & $\mathrm{CP} 2$ & & & \\
\hline & P. sigmoides & CP1 & $\frac{b}{a}$ & & $\begin{array}{c}9 \mathrm{R}-\mathrm{CC} \rightarrow 10 \mathrm{R}-1,120 \\
10 \mathrm{R}-2,68 \rightarrow 10 \mathrm{R}-2,142\end{array}$ \\
\hline
\end{tabular}

Figure 3. Distribution of Paleogene sediments from Leg 123 based on calcareous nannofossil biostratigraphy.

1967) and denote an age of early Paleocene (Fig. 3). Early Paleocene sediments continue in Samples 123-766A-9R-5, 109-111 $\mathrm{cm}$, through $-9 \mathrm{R}-4,109-111 \mathrm{~cm}$, and were placed in Zone CP2 (Chiasmolithus danicus Zone) based on the presence of Chiasmolithus danicus. Samples 123-766A-9R-3, 109-111 cm, through $-9 \mathrm{R}-1,109-111 \mathrm{~cm}$, were placed in undifferentiated Zones CP2CP3 (Chiasmolithus danicus/Ellipsolithus macellus zones) and are early Paleocene in age.

A thick, late Paleocene-early Eocene sediment section that includes a complete Paleocene/Eocene boundary can be found first in Sample 123-766A-8R-CC and continues through Sample 123-766A-4R-CC. Samples 123-766A-8R-CC through -8R-2, 31-34 cm, were placed in Zone CP6 (Discoaster mohleri Zone) based on the presence of D. mohleri. Zone CP7 (Discoaster nobilis Zone), and this zone was assigned to sediments in Sample 123-766A-8R-1, 92-95 cm, based on the presence of $D$. nobilis. Samples 123-766A-7R-CC through $-6 \mathrm{R}-6,114-116 \mathrm{~cm}$, were placed in Zone CP8 (Discoaster multiradiatus Zone) based on the presence of D. multiradiatus with a subdivision to Subzone CC8b
(Camplyosphaera eodela Subzone) first occurring in Sample 123-766A-6R-CC based on the first appearance of $C$. eodela. Zone CP9 (Discoaster diastypus Zone) was assigned to sediments in Samples 123-766A-6R-5, 100-105 cm, through -4R-CC based on the presence of $D$. diastypus, but could not be differentiated into Subzones CP9a and CP9b because of the absence of Tribrachiatus contortus.

The completeness of the late Paleogene-early Eocene record and the high diversity of nannofossil assemblages make this interval unique. Members of the genera Fasciculithus, Toweius, and Chiasmolithus are particularly diverse. High assemblage diversities may result from the mid-latitude position of this site during this time. In modern waters, the highest diversity of coccolithophorids occurs in mid-latitude (between $15^{\circ}$ and $30^{\circ}$ ) zones of upwelling (Okada and Honjo, 1973). This evidence combined with the absence of certain ecologically controlled marker species (such as Tribrachiatus Perch-Nielsen, 1985) further support an interpretation of a mid-latitude position for Site 766 during this time. 
Table 4. Paleogene calcareous nannofossils from Site $\mathbf{7 6 6 .}$

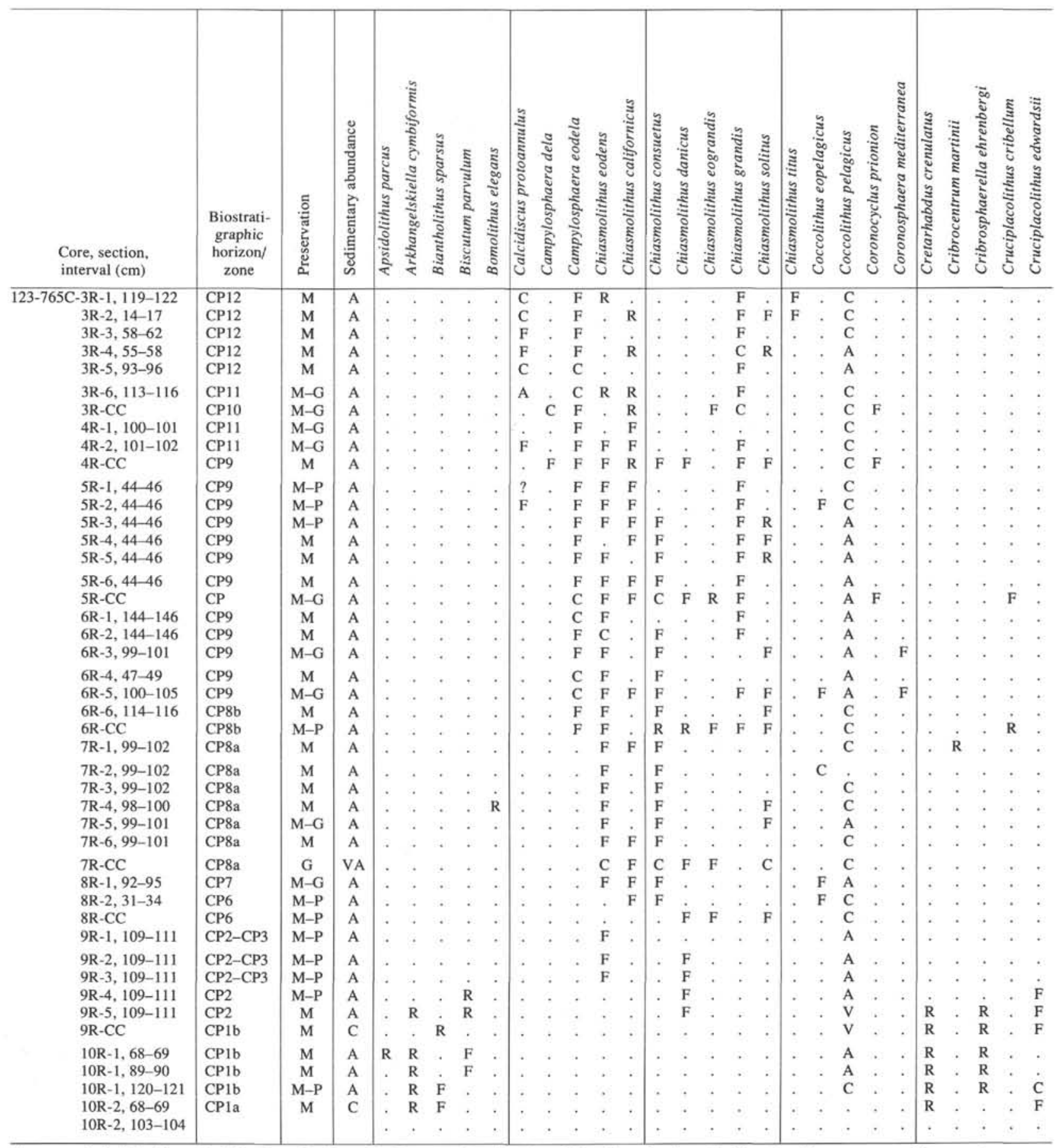

Key to symbols: $\mathrm{RE}=$ Reworked; $\mathrm{R}=$ Rare; $\mathrm{F}=\mathrm{Few} ; \mathrm{C}=$ Common; $\mathrm{A}=\mathrm{Abundant} ; \mathrm{VA}=$ Very abundant; ? = Questionable presence; , = Not present.

Early Eocene sediments continue in Samples 123-766A-4R-2, $101-102 \mathrm{~cm}$, through $-3 \mathrm{R}-6,113-116 \mathrm{~cm}$, and these were placed in Zone CP11 (Discoaster lodoensis Zone) based on the presence of D. lodoensis. Samples 123-766A-3R-5, 93-96 cm, through $-3 \mathrm{R}-1,119-122 \mathrm{~cm}$, were placed in Zone CP12a (Discoasteroides kuepperi Subzone) based on the presence of Discoaster sublodoensis and D. kuepperi and are of early Eocene age. A significant unconformity overlies Core 123-766A-3R, with late/early Pliocene sediments found in Sample 123-766A-2R-CC. Accord- ing to Berggren et al. (1985), this unconformity spans at least $24.1 \mathrm{Ma}$.

\section{DISCUSSION AND CONCLUSIONS}

Calcareous nannofossil biostratigraphy of core material obtained from Holes $765 \mathrm{C}$ and 766A during Leg 123 revealed thick and relatively complete sections of Upper Cretaceous-Paleogene sediments in each hole. Hole $765 \mathrm{C}$ has a nearly complete AlbianOligocene interval that contains abundant and generally moderate 


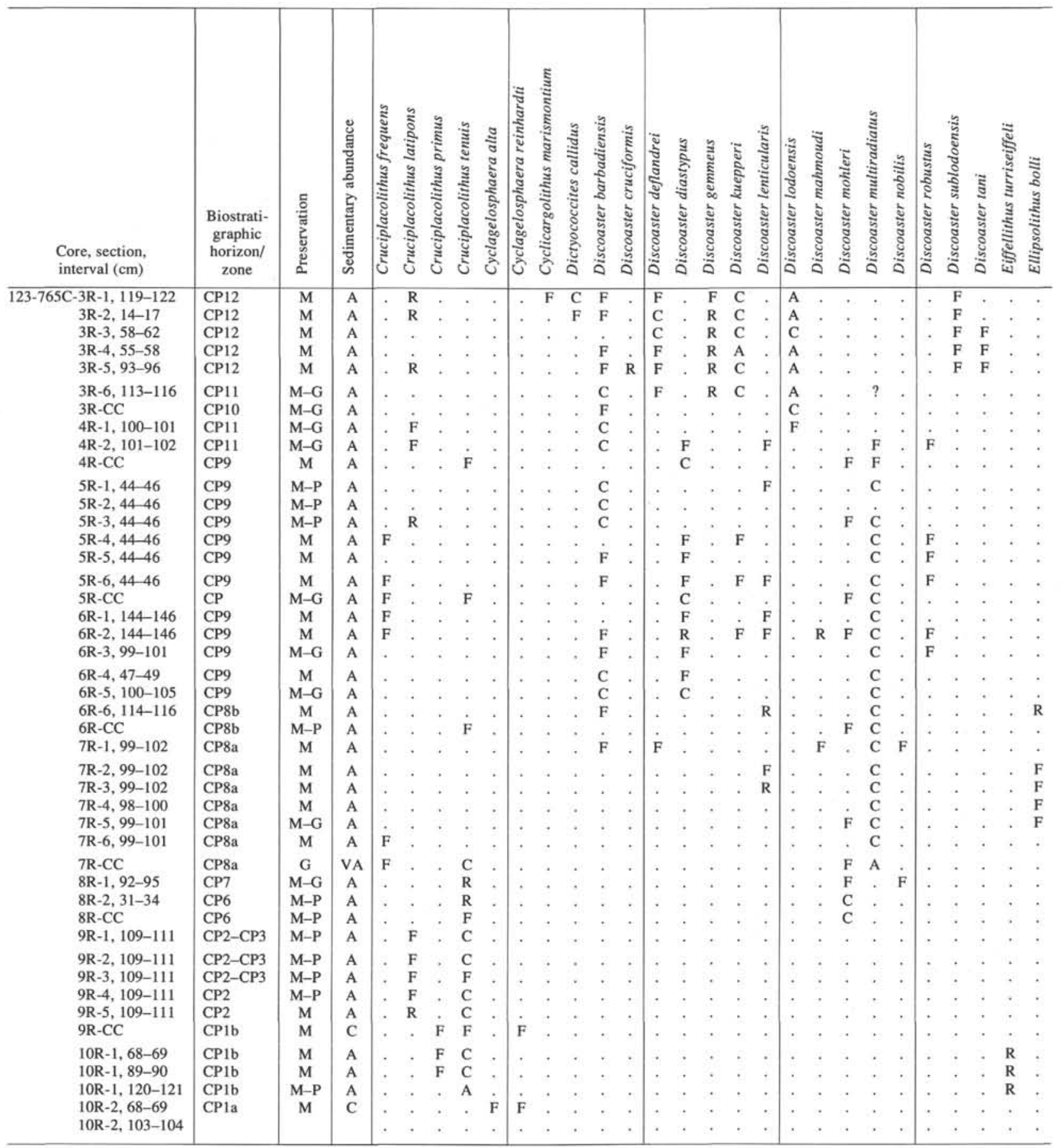

to well-preserved nannofossils that allow one to correlate accurately. Although turbidite deposition dominates in the upper part of this hole, reworking is minimal below the Miocene section. A particularly thick Aptian section exists in this hole that spans 13 cores. Hole $766 \mathrm{~A}$ has a thick and relatively complete Albianlower Eocene interval. The hemipelagic to pelagic depositional environment of the sediments found in this hole allowed for generally good preservation of diverse nannofossil assemblages and nominal reworking.

Several confidently interpreted unconformities occur in sections of each hole. In the Upper Cretaceous section of Hole 765C, the most confidently interpreted unconformities span much of the Santonian, lower Campanian, and Maestrichtian. Paleogene unconformities occur in the upper Eocene and lower Oligocene. Following the time scale data of Berggren et al. (1985), the late Eocene unconformity spans at least $12 \mathrm{Ma}$, while the early Oligocene unconformity spans at least $8.5 \mathrm{Ma}$. In the Upper Cretaceous of Hole 766A, confidently interpreted unconformities span much of the Coniacian, lower Campanian, and Maestrichtian. The most significant Paleogene unconformity in this hole spans the upper Eocene to the lower Pliocene, and, following Berggren et al. (1985), spans at least 24.1 Ma. The chronostratigraphic position 
Table 4 (continued).

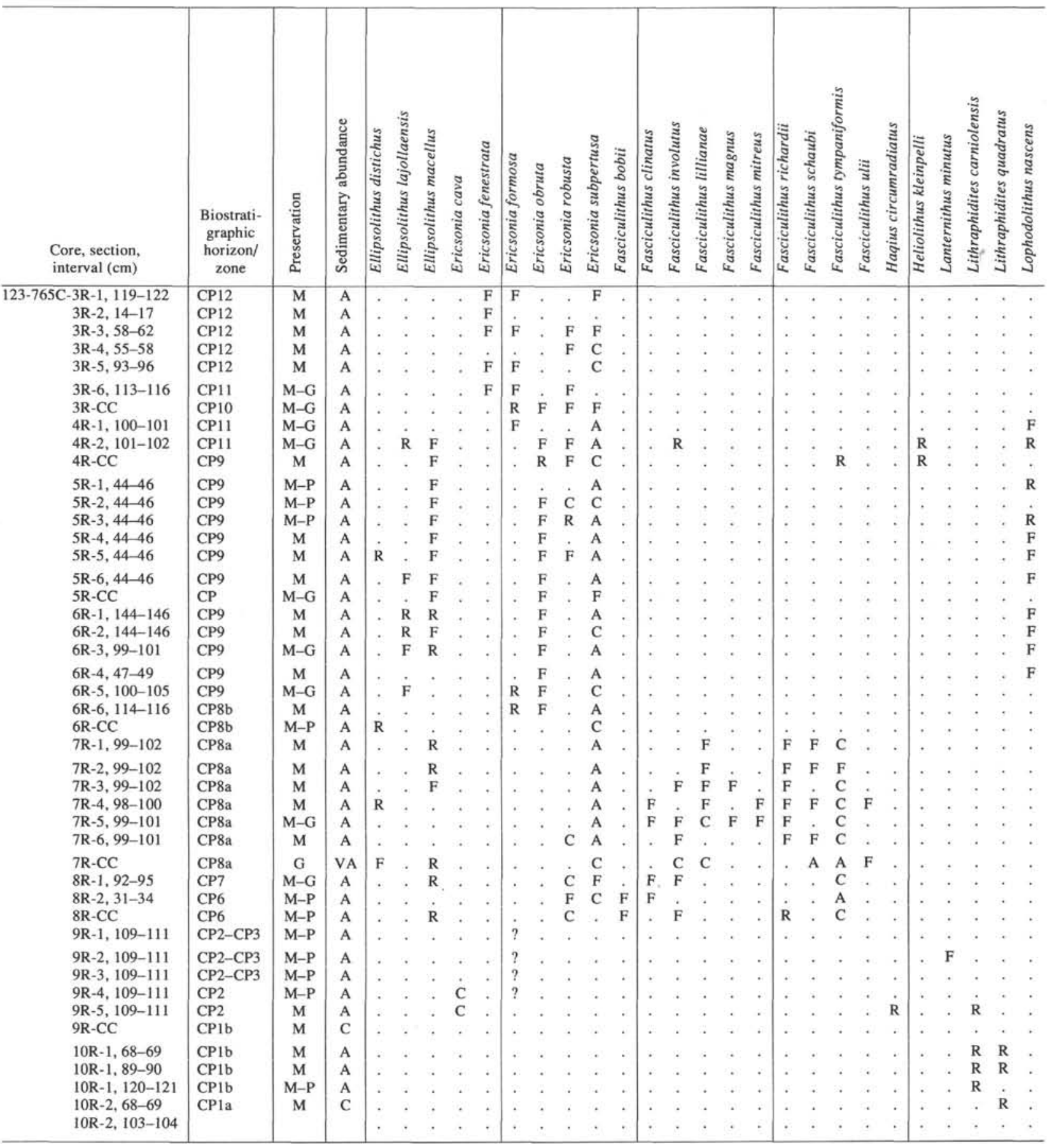

and length of all these unconformities may have considerable significance for reconstructing the sedimentary history and for interpreting the paleoceanography of this region.

Calcareous nannofossil assemblages found in the Upper Cretaceous-Paleogene section of Hole $765 \mathrm{C}$ and the Upper Cretaceous section of Hole 766A are fairly standard and do not differ significantly from assemblages typical of established, low-latitude zonations, such as those discussed by Sissingh (1977) and Okada and Bukry (1980). Extensive reworking of nannofossil species occurs in the lower Oligocene and near the Cretaceous/Paleogene boundary of Hole $765 \mathrm{C}$.
Although the nannofossil assemblages from the thick and complete Paleogene/Eocene section of Hole 766A were correlated successfully using the low-latitude zonation of Okada and Bukry (1980), biostratigraphic resolution was reduced by the absence of the marker taxon Tribrachiatus contortus and the resultant inability to differentiate Subzones CP9a and CP9b. Paradoxically, assemblages are diverse in this interval and contain a large number of species in the genera Fasciculithus, Toweius, and Chiasmolithus. Specimens of less typical genera, such as Neocrepidolithus and Neochiastozygus, also are commonly present. These data (absence of ecologically restricted marker species and high as- 
Table 4 (continued).

\begin{tabular}{|c|c|c|c|c|c|c|c|c|c|c|c|c|c|c|c|c|c|c|c|c|c|c|c|c|c|c|c|c|}
\hline $\begin{array}{l}\text { Core, section, } \\
\text { interval }(\mathrm{cm})\end{array}$ & $\begin{array}{c}\text { Biostrati- } \\
\text { graphic } \\
\text { horizon/ } \\
\text { zone }\end{array}$ & 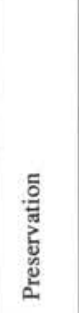 & 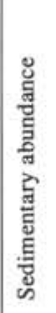 & 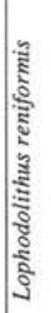 & 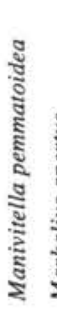 & 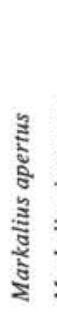 & 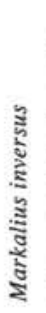 & 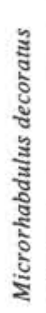 & 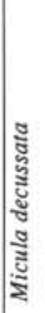 & 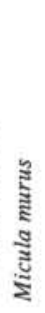 & 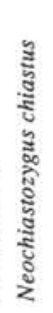 & 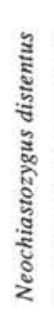 & 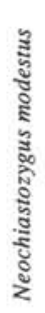 & 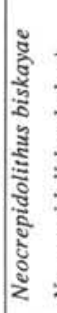 & 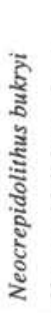 & 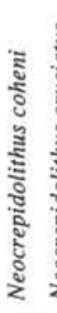 & 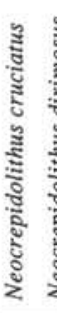 & 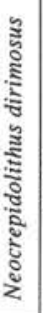 & 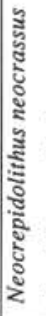 & 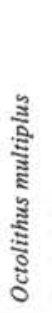 & 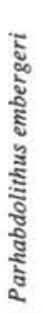 & 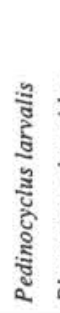 & 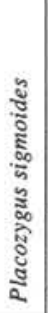 & 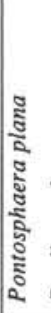 & 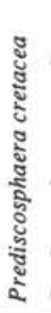 & 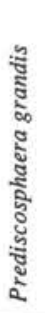 & 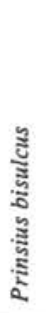 & 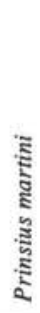 \\
\hline $123-765 \mathrm{C}-3 \mathrm{R}-1,119-122$ & CP12 & M & A & . & . & . & $\mathbf{R}$ & . & . & . & . & &. & . & $F$ & . & . & . & . & . & . & . & . & . & . & . & . & \\
\hline $3 \mathrm{R}-2,14-17$ & CP12 & M & A & . & . & . & $\mathbf{R}$ & . & . & . & . & & . & . & F & . & . & . & . & . & . & $\mathrm{R}$ & . & . & . & . & . & . \\
\hline $3 R-3,58-62$ & CP12 & M & A & . & & . & $\mathrm{F}$ & . & . & . & . & $\mathbf{R}$ & . & . & . & . & . & . & . & . & . & . & . & . & . & . & . & . \\
\hline $3 R-4,55-58$ & CP12 & M & A & . & . & . & . & . & . & . & . & . & . & & $\mathrm{F}$ & . & . & . & . & . & . & . & . & . & . & . & . & . \\
\hline $3 R-5,93-96$ & CP12 & M & A & . & . & . & . & $\mathrm{R}$ & . & . & . & . & . & & $\mathrm{F}$ & . & . & . & . & . & . & · & . & . & & • & $\cdot$ & . \\
\hline $3 R-6,113-116$ & CP11 & $M-G$ & A & . & . & . & $\mathbf{R}$ & . & . & . & . & . & . & . & $\mathrm{F}$ & . & . & . & . & . & . & . & . & . & . & . & . & . \\
\hline $3 \mathrm{R}-\mathrm{CC}$ & CP10 & $M-G$ & A & . & , & . & & . & . & - & . & $\dot{0}$ & . & . & F & . & . & . & . & . & . & . & . & . & . & . & . & . \\
\hline $4 \mathrm{R}-1,100-101$ & CP11 & M-G & A & . & . & $\mathrm{R}$ & $\mathrm{F}$ & . & . & . & . & $\mathrm{R}$ & . & . & $\mathrm{F}$ & . & . & . & . & . & . & . & . & . & . & . & . & . \\
\hline $4 \mathrm{R}-2,101-102$ & CP11 & $M-G$ & A & & . & . & $\mathrm{F}$ & . & . & . & . & $\mathbf{R}$ & . & & F & . & . & . & . & . & . & . & $\mathrm{F}$ & . & . & . & . & . \\
\hline $4 \mathrm{R}-\mathrm{CC}$ & CP9 & M & A & $\mathbf{R}$ & . & . & R & . & . & . & . & . & . & R & $\mathrm{F}$ & . & . & . & . & . & . & . & . & . & . & . & F & . \\
\hline $5 \mathrm{R}-1,44-46$ & CP9 & M-P & A & . & . & . & $\mathrm{F}$ & . & . & . & . & $\mathrm{R}$ & . & . & F & . & . & . & . & . & . & . & . & . & . & . & . & . \\
\hline $5 \mathrm{R}-2,44-46$ & CP9 & M-P & A & . & . & . & $\mathrm{F}$ & . & . & . & . & . & - & - & $\mathrm{F}$ & . & . & . & . & . & . & . & . & . & . & . & . & . \\
\hline $5 R-3,44-46$ & CP9 & M-P & A & . & . & . & $\mathrm{F}$ & . & . & . & . & & . & . & $\mathrm{F}$ & . & . & . & . & . & . & . & . & . & . & . & . & . \\
\hline $5 R-4,44-46$ & CP9 & M & A & . & . & . & . & - & . & . & . & $\mathbf{R}$ & . & . & F & . & . & . & . & . & . & . & . & . & . & . & . & . \\
\hline $5 R-5,44-46$ & CP9 & M & A & . & . & . & R & . & . & . & . & $\mathbf{R}$ & . & . & F & . & . & . & . & . & . & . & . & . & . & . & . & . \\
\hline $5 R-6,44-46$ & CP9 & M & A & . & . & . & $\mathbf{R}$ & . & . & . & . & . & . & . & F & . & . & . & . & . & . & . & $\mathrm{F}$ & . & . & . & . & . \\
\hline 5R-CC & CP & M-G & A & . & . & $\mathbf{R}$ & . & . & . & . & . & $\dot{0}$ & . & $\mathbf{R}$ & F & . & . & . & . & . & . & . & $\dot{r}$ & . & . & . & . & . \\
\hline $6 \mathrm{R}-1,144-146$ & CP9 & M & A & . & . & . & . & . & . & . & . & $\mathbf{R}$ & . & . & F & . & . & . & . & . & . & . & $\mathrm{F}$ & . & . & . & . & . \\
\hline $6 \mathrm{R}-2,144-146$ & CP9 & M & A & . & . & . & $\mathrm{R}$ & . & . & . & . & $\dot{x}$ & . & . & $\mathbf{R}$ & . & . & . & . & . & . & . & $\mathrm{F}$ & . & . & . & . & . \\
\hline $6 \mathrm{R}-3,99-101$ & CP9 & $M-G$ & A & . & . & . & $\mathbf{R}$ & . & . & . & . & $\mathrm{F}$ & . & . & $\mathrm{F}$ & . & . & . & . & . & . & . & $\mathrm{F}$ & . & . & . & . & . \\
\hline $6 \mathrm{R}-4,47-49$ & CP9 & $\mathrm{M}$ & A & . & . & $\dot{c}$ & $\dot{\dot{x}}$ & . & . & . & . & $\mathrm{F}$ & . & . & F & . & . & . & . & . & . & . & $\mathrm{F}$ & . & . & . & . & . \\
\hline $6 \mathrm{R}-5,100-105$ & СР9 & $M-G$ & A & & . & $\mathrm{F}$ & & . & . & . & . & & . & . & F & & . & . & . & . & . & . & & . & & . & . & . \\
\hline $6 R-6,114-116$ & CP8b & M & A & . & . & . & R & . & . & . & . & $\mathbf{R}$ & . & . & $\mathrm{F}$ & . & . & . & . & . & . & . & F & . & . & . & . & . \\
\hline 6R-CC & CP8b & M-P & A & & . & . & $\mathbf{R}$ & . & . & . & . & . & . & . & $\mathrm{F}$ & . & . & . & . & . & . & . & & . & . & . & F & . \\
\hline $7 \mathrm{R}-1,99-102$ & CP8a & M & A & . & . & . & $\mathrm{R}$ & . & . & . & . & . & . & . & $\mathrm{F}$ & . & . & . & . & . & . & . & $\mathrm{F}$ & . & . & . & . & . \\
\hline $7 \mathrm{R}-2,99-102$ & CP8a & M & A & . & . & . & $\mathbf{R}$ & . & . & . & . & . & . & $\mathbf{R}$ & $\mathrm{F}$ & . & . & . & . & . & . & . & $\mathrm{F}$ & . & . & . & . & . \\
\hline $7 \mathrm{R}-3,99-102$ & CP8a & M & A & . & . & . & $\mathrm{R}$ & . & . & . & . & . & . & $\mathrm{R}$ & $\mathrm{R}$ & . & . & . & . & . & . & . & & . & . & . & . & . \\
\hline $7 R-4,98-100$ & CP8a & $\mathrm{M}$ & A & & . & . & . & . & . & . & . & . & . & $\mathrm{F}$ & $\mathrm{R}$ & . & . & . & . & . & . & . & $\mathbf{R}$ & . & & . & . & . \\
\hline 7R-5, 99-101 & CP8a & $M-G$ & A & . & . & . & . & . & . & . & . & . & . & . & $\mathrm{F}$ & . & . & . & . & . & . & . & $\mathrm{F}$ & . & . & . & . & . \\
\hline 7R-6, 99-101 & CP8a & M & A & . & . & . & $\mathrm{F}$ & . & . & . & . & . & . & . & $\mathrm{F}$ & . & . & . & . & . & . & . & F & . & . & . & . & . \\
\hline 7R-CC & CP8a & G & VA & . & . & . & . & . & . & . & . & . & . & . & $\mathrm{F}$ & . & . & . & . & . & . & . & $\mathbf{R}$ & . & . & . & & . \\
\hline $8 \mathrm{R}-1,92-95$ & CP7 & $M-G$ & A & . & . & . & . & . & . & . & . & . & . & $\mathbf{R}$ & $\mathbf{R}$ & . & . & . & . & . & . & . & $\mathrm{R}$ & 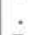 & . & . & $\mathbf{R}$ & . \\
\hline $8 R-2,31-34$ & CP6 & M-P & A & . & . & $\mathrm{F}$ & . & . & . & . & $\mathrm{R}$ & . & . & $\mathbf{R}$ & $\mathbf{R}$ & . & . & . & . & . & . & . & & $\mathbf{R}$ & . & . & . & . \\
\hline $8 R-C C$ & CP6 & M-P & A & . & . & . & . & . & . & . & . & . & . & . & $\mathbf{R}$ & . & . & . & . & . & . & . & F & . & . & . & & . \\
\hline $9 \mathrm{R}-1,109-111$ & $\mathrm{CP} 2-\mathrm{CP} 3$ & M-P & A & . & . & $\mathrm{R}$ & $\mathrm{F}$ & . & . & . & . & . & . & . & . & . & $\mathbf{R}$ & . & . & . & . & . & C & . & . & . & F & . \\
\hline 9R-2, 109-111 & $\mathrm{CP} 2-\mathrm{CP} 3$ & M-P & A & . & . & . & $\mathrm{R}$ & . & . & . & F & . & . & . & . & . & . & . & $\mathbf{R}$ & . & . & . & C & . & . & . & F & . \\
\hline $9 \mathrm{R}-3,109-111$ & $\mathrm{CP} 2-\mathrm{CP} 3$ & M-P & A & & . & $\mathrm{R}$ & F & . & . & . & . & . & . & . & . & & R & . & . & & . & & & . & . & . & & F \\
\hline $9 \mathrm{R}-4,109-111$ & CP2 & M-P & A & . & . & $\mathrm{R}$ & $\mathrm{F}$ & & & $\cdot$ & . & . & . & . & . & . & . & . & . & F & . & . & $\mathrm{C}$ & . & $\mathbf{R}$ & . & F & . \\
\hline $9 \mathrm{R}-5,109-111$ & $\mathrm{CP} 2$ & M & A & : & . & $\mathrm{F}$ & $\mathrm{F}$ & $\mathrm{R}$ & $\mathrm{R}$ & . & . & . & . & . & . & $\mathbf{R}$ & . & . & . & F & $\mathbf{R}$ & & C & . & $\mathbf{R}$ & . & F & . \\
\hline 9R-CC & CPIb & M & C & . & . & . & $\mathrm{F}$ & . & $\mathrm{R}$ & . & . & . & . & . & . & R & . & . & . & . & . & . & C & . & R & . & . & . \\
\hline $10 \mathrm{R}-1,68-69$ & CP1b & M & A & . & $R$ & $\mathrm{~F}$ & F & R & R & . & . & . & . & . & . & . & C & . & . & F & $\mathbf{R}$ & & & . & $\mathrm{R}$ & $\mathbf{R}$ & . & $\mathrm{F}$ \\
\hline $10 \mathrm{R}-1,89-90$ & CP1b & $\mathrm{M}$ & A & . & . & $\mathrm{F}$ & $\mathrm{F}$ & $\mathbf{R}$ & $\mathrm{R}$ & & . & . & 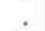 & . & . & . & $\mathrm{F}$ & & . & . & . & . & $\mathrm{C}$ & . & $\mathbf{R}$ & $\mathbf{R}$ & . & $\mathrm{F}$ \\
\hline $10 \mathrm{R}-1,120-121$ & CPIb & M-P & A & . & . & $\mathrm{F}$ & C & $\mathbf{R}$ & $\mathrm{R}$ & & . & . & $\mathbf{R}$ & . & . & $\mathrm{F}$ & $\mathrm{F}$ & $\mathrm{F}$ & . & . & . & . & $\mathrm{C}$ & . & $\mathbf{R}$ & . & . & . \\
\hline $10 \mathrm{R}-2,68-69$ & CP1a & $\mathrm{M}$ & $\mathrm{C}$ & . & . & . & $\mathrm{C}$ & . & $\mathrm{R}$ & $\mathrm{R}$ & . & . & . & . & . & $\mathrm{F}$ & . & . & . & . & . & . & A & . & $\mathrm{R}$ & . & . & . \\
\hline $10 \mathrm{R}-2,103-104$ & & & & . & . & . & . & . & . & . & . & . & . & . & . & . & . & . & . & . & . & . & . & . & . & . & . & . \\
\hline
\end{tabular}

semblage diversity) suggest that this site may have had a mid-latitude position during the upper Paleocene-lower Eocene, which is supported by the following evidence:

1. According to Okada and Honjo (1973), in modern waters the highest diversity of coccolithophorids occurs between $15^{\circ}$ and $30^{\circ}$ in mid-latitude zones of upwelling.

2. Perch-Nielsen (1985) indicated that members of the genus Tribrachiatus are generally found only in low paleolatitudes.

Other shipboard nannofossil biostratigraphic data for Site 766 (Ludden, Gradstein, et al., 1990) suggest a mid-latitude position during this time, including (1) the successful application of the mid-latitude Subzone CN10d (Amaurolithus delicatus Bukry, 1981 ) in Neogene sediments from Hole 766A, (2) the persistence of Dictyococcites spp. into the Neogene, and (3) the absence of Tribrachiatus spp. in the lower Eocene.

\section{ACKNOWLEDGMENTS}

I would first like to thank the Ocean Drilling Program and the United States Science Advisory Committee (USSAC) for providing financial support while completing this project. The facilities for generating and managing the data of this study were provided by the Geology Department of the University of Nebraska and are 
Table 4 (continued).

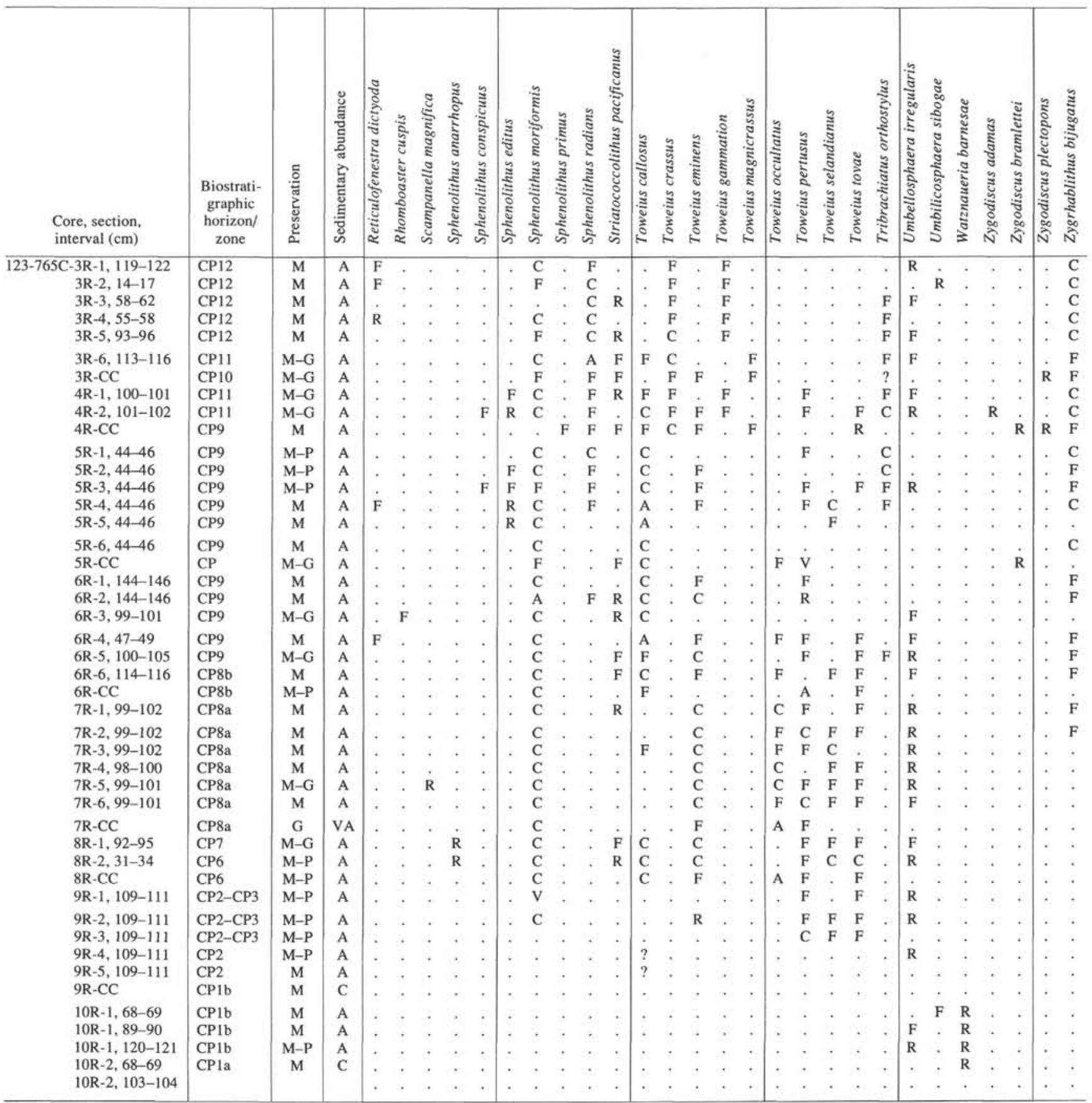

gratefully acknowledged. Thanks also are extended to the Geology Department of the University of Papua New Guinea for providing support during production of the manuscript.

\section{REFERENCES}

Berggren, W. A., Kent, D. V., and Flynn, J. J., 1985. Jurassic to Paleogene: Part 2, Paleogene geochronology and chronostratigraphy. In Snelling, N. J. (Ed.), The Chronology of the Geologic Record: New York (Blackwell Scientific Publications), 141-195.

Hay, W. W., 1970. Calcareous nannofossils from cores recovered on Leg 4. In Bader, R. G., Gerard, R. D., et al., Init. Repts. DSDP, 4: Washington, D.C. (U.S. Govt. Printing Office), 445-501.
Hay, W. W. and Mohler, D., 1967. Calcareous nannoplankton from early Tertiary rocks at Pont Labau, France, and Paleocene-Eocene correlations. J. Paleontol., 41:1505-1541.

Ludden, J. N., Gradstein, F. M., et al., 1990. Proc. ODP, Init. Repts, 123: College Station, TX (Ocean Drilling Program).

Moran, M. J., and Watkins, D. K., 1988. Oligocene calcareous nannofossil biostratigraphy from Leg 101, Site 628, Little Bahama Bank Slope. In Austin, J. A., Jr., Schlager, W., Palmer, A. A., et al., Proc. ODP, Sci. Results, 101: College Station, TX (Ocean Drilling Program), 87-103.

Okada, H., and Honjo, S., 1973. The distribution of oceanic coccolithophorids in the Pacific. Deep-Sea Res., 20:355-374.

Okada, H., and Bukry, D., 1980. Supplementary modification and introduction of code numbers to the "low-latitude coccolith biostrati- 
graphic zonation" (Bukry 1973; 1975). Mar. Micropaleontol., 5:321325.

Perch-Nielsen, K., 1979. Calcareous nannofossils from the Cretaceous between the North Sea and the Mediterranean. IUGS Ser. A, 6:223-272. 1985. Cenozoic calcareous nannofossils. In Bolli, H. M., Saunders, J. B., and Perch-Nielsen, K. (Eds.), Plankton Stratigraphy: Cambridge (Cambridge University Press), 427-554.

Sissingh, W., 1977. Biostratigraphy of Cretaceous calcareous nannoplankton. Geol. Minjbouw., 56(1):37-65.
Watkins, D. K., and Bowdler, J. L., 1984. Cretaceous calcareous nannofossils from Deep Sea Drilling Project Leg 77, Southeast Gulf of Mexico. In Buffler, R. T., Schlager, W., et al., Init. Repts. DSDP, 77: Washington, D.C. (U.S. Govt. Printing Office), 649-674.

Date of initial receipt: 15 June 1990

Date of acceptance: 12 September 1991

Ms 123B-118 